\title{
Spectral properties of eight near-Earth asteroids ${ }^{\star}$
}

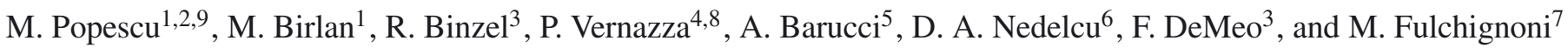 \\ ${ }^{1}$ Institut de Mécanique Céleste et de Calcul des Éphémérides (IMCCE), Observatoire de Paris, 77 avenue Denfert-Rochereau, \\ 75014 Paris Cedex, France \\ e-mail: mpopescu@imcce.fr \\ 2 Polytechnic University of Bucharest, Faculty of Applied Sciences, Department of Physics, Bucharest, Romania \\ 3 Department of Earth, Atmospheric, and Planetary Sciences, Massachusetts Institute of Technology, 77 Massachusetts Avenue, \\ Cambridge, MA 02139, USA \\ ${ }^{4}$ Laboratoire d'Astrophysique de Marseille, Université de Provence-CNRS, 38 rue Frédéric Joliot-Curie, 13388 Marseille Cedex 13 , \\ France \\ ${ }^{5}$ LESIA, Observatoire de Paris, 5 place Jules Janssen, 92195 Meudon Principal Cedex, France \\ 6 Astronomical Institute of the Romanian Academy, 5 Cuţitul de Argint, 040557 Bucharest, Romania \\ 7 Université Paris Diderot - Paris 7, 4 rue Elsa Morante, 75013 Paris, France \\ 8 European Southern Observatory, K. Schwarzschild-Str. 2, 85748 Garching, Germany \\ 9 Bucharest Astroclub, B-dul LascarCatargiu 21, sect 1, Bucharest, Romania
}

Received 20 April 2011 / Accepted 21 July 2011

\begin{abstract}
Context. Near-Earth objects are among the most accessible bodies in the solar system in terms of the spacecraft propulsion requirements to reach them. The choice of targets and the planning of space missions are based on high quality ground-based science.

Aims. The knowledge of the ensemble of physical parameters for these objects, including their composition, is a critical point in defining any mission scientific objectives. Determining the physical properties of near-Earth asteroids (NEAs) is also possible from the ground by analyzing spectroscopy at both visible and infrared wavelengths.

Methods. We present spectra of eight NEAs (1917, 8567, 16960, 164400, 188452, 2001 SG286, and 2010 TD54) obtained using the NASA telescope IRTF equipped with the spectro-imager SpeX. The observations were performed in the $0.8-2.5 \mu \mathrm{m}$ spectral region using the low resolution mode of the spectrograph. We completed the taxonomic classification using the Bus-DeMeo taxonomy. We analyzed the spectra by comparing them to meteorite spectra from the Relab database using a $\chi^{2}$ approach. For the S-type asteroids of our sample, the band centers and BAR were calculated. We also attempted to interpret our data using a space-weathering model. Results. The taxonomic classification of five objects was reviewed and we assigned a corresponding type to the other three asteroids that were not classified before. We found that (1917) Cuyo, (8567) 1996 HW1, (16960) 1998 QS52, (188452) 2004 HE62, and 2010 TD54 are in the S-complex. We achieved a good matching of our S-type asteroids with the spectra of ordinary chondrites meteorites. The asteroid (5620) Jasonwheeler was found to have a NIR spectrum similar to carbonaceous chondrite meteorites. Thus, our results confirm its primitive properties obtained in several other spectral intervals.
\end{abstract}

Key words. minor planets, asteroids: general - methods: observational - techniques: spectroscopic

\section{Introduction}

Asteroids are leftovers from the formation of the solar system and studying their properties in detail will allow us to constrain more reliably the formation and evolution of our solar system.

There are more than 500000 known asteroids, most of them belonging to the main belt. Owing to some mechanisms, which are still the subject of dynamical studies, some of these objects have migrated into the inner part of the solar system (Morbidelli et al. 2002). These are near-Earth asteroids (denoted NEAs), small bodies of the solar system with perihelion distances $q \leq$ 1.3 AU and aphelion distances $Q \geq 0.983 \mathrm{AU}$, whose orbits approach or intersect the Earth orbit. Depending on their orbital parameters, NEAs are divided into Apollos ( $a \geq 1.0 \mathrm{AU}$; $q \leq 1.016 \mathrm{AU})$, Athens ( $a<1.0 \mathrm{AU} ; Q \geq 0.983 \mathrm{AU})$, and Amors $(1.016<q<1.3 \mathrm{AU})$.

Potentially hazardous asteroids (PHAs) are currently defined based on parameters that measure the asteroid's potential

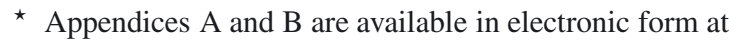
http: //www . aanda.org to make threatening close approaches to the Earth. All asteroids with an Earth minimum orbit intersection distance (MOID) smaller than $0.05 \mathrm{AU}$ and an absolute magnitude $(H)$ of 22.0 or brighter are considered PHAs (Milani et al. 2000).

One of the most important aspects related to the NEAs is their accessibility to be investigated by spacecrafts. This enables their scientific study and the detailed assessment of their future use as space resources. Several programs for space exploration of these objects (Marco Polo-R, OSIRIS-REx, Hayabusa2) are now under study around the World. The choice of targets and the planning of space exploration are based on strong groundbased science. Thus, the knowledge of the ensemble of physical parameters of objects and their composition is a critical point in defining the mission scientific objectives.

A major scientific goal of studies of the NEA population is their global characterization in terms of spectral trends, relating the spectral data to the laboratory measurements. Given the dominance of S-type asteroids among the NEA population, and the abundance of ordinary chondrites (OC) among the meteorites, it has been generally and widely assumed that they are connected, 
Table 1. Log of asteroids observations.

\begin{tabular}{lccccccccc}
\hline \hline Asteroid & Date (UT) & $V$ & $\Phi\left(^{\circ}\right)$ & $r(\mathrm{UA})$ & Airmass & ITime(s) & Cycles & Solar analogue & Airmass \\
\hline (1917) Cuyo & $2008 / 08 / 27.637$ & 14.6 & 66.0 & 1.105 & 1.038 & 120 & 6 & BD+41 309 & 1.141 \\
(5620) Jasonwheeler & $2009 / 05 / 04.569$ & 16.5 & 20.9 & 1.345 & 1.344 & 120 & 6 & HD 154716 & 1.240 \\
(8567) 1996 HW1 & $2008 / 08 / 27.543$ & 12.9 & 28.8 & 1.143 & 1.099 & 60 & 13 & HD 217577 & 1.213 \\
(16960) 1998 QS52 & $2008 / 08 / 27.588$ & 16.9 & 30.0 & 1.784 & 1.105 & 120 & 13 & HD 27834 & 1.083 \\
(164400) 2005 GN59 & $2008 / 08 / 27.472$ & 16.2 & 25.2 & 1.244 & 1.024 & 120 & 2 & BD+28 3198 & 1.410 \\
(188452) 2004 HE62 & $2008 / 08 / 27.404$ & 16.7 & 60.8 & 1.109 & 1.513 & 120 & 12 & BD+28 3198 & 1.401 \\
2001 SG286 & $2009 / 05 / 19.594$ & 16.7 & 102.0 & 1.006 & 1.962 & 120 & 2 & HD 216516 & 1.742 \\
2010 TD54 & $2010 / 10 / 12.303$ & 15.5 & 17.3 & 1.000 & 1.252 & 120 & 8 & L115-271 & 1.092 \\
\hline
\end{tabular}

Notes. Asteroid designations, date of observation with the fraction of the day for the mid time of the observation, the apparent magnitude, the phase angle, the heliocentric distance, the airmass at the mean UT of each observation, the integration time for each spectrum (ITime), and the number of cycles are presented. The last two columns describe the solar analogs used for data reduction, as well as their airmass at the moment of observations.

and that NEAs are the most probably parent bodies of meteorites (Vernazza et al. 2008; de León et al. 2010). Vernazza et al. (2008) reported that about two-thirds of near-Earth asteroids in their sample of 38 objects have spectral properties quantitatively similar to the LL meteorites.

Statistical analysis of spectral data allows the construction of taxonomies and taxonomic classes. These analyzes represent a first step in studies of comparative planetology, which permit us to characterize the specific mineralogy of each class that they identify. The new taxonomy for asteroids obtained by DeMeo et al. (2009) underlines the importance of both visible and nearinfrared spectral data to determining the asteroid statistics. This is quite understandable while the spectral data of regoliths on the asteroid's surface are of the $0.4-3.6 \mu \mathrm{m}$ spectral region ${ }^{1}$. With few exceptions, this spectral region is accessible from the ground, the atmosphere being transparent at these wavelengths. This new Bus-DeMeo taxonomy, based on the spectra of more than 310 objects, defines 24 classes.

Irradiation by cosmic and solar wind ions, as well as bombardment by interplanetary dust particles (micro-meteorites) produce relevant surface modifications to airless bodies of the solar system. These processes are known as space weathering (Hapke 2001). The most affected by this alteration are silicaterich objects, for which a progressive darkening and reddening of the solar reflectance spectra appear in the $0.2-2.7 \mu \mathrm{m}$ spectral region (Hapke 2001). Space weathering processes can explain the spectral differences between the ordinary chondrite meteorites and their presumed parent bodies - the S-type asteroids (Pieters et al. 2000). Vernazza et al. (2009) demonstrate the necessity to take the composition into account when evaluating weathering effectiveness. The laboratory experiments show that solarwind ion implantation is the most rapid of several competing processes (Brunetto et al. 2006; Vernazza et al. 2009; Loeffler et al. 2009).

On the basis of laboratory experiments, Brunetto et al. (2006) describe the spectral effects of solar wind irradiation by an exponential continuum in terms of the ratio of weathered spectra to unweathered spectra. Since ion-induced spectral reddening is related to the formation of displacements, they correlated the model with a damage parameter of the surface given as the number of displacements per $\mathrm{cm}^{2}$.

In this paper, we present spectroscopic results for eight NEAs in the $0.8-2.5 \mu \mathrm{m}$ spectral region. The asteroids were observed during several runs between 2008 and 2010 to study NEA physical properties, and are subsidiary to the potential targets of spacecraft missions. In Sect. 2, the details related to observation

\footnotetext{
1 We refer here only to the reflectance spectra.
}

methods and the steps followed for data reduction are given. We modeled and interpreted the acquired spectra using different techniques with the goal of achieving basic interpretations regarding of the composition and physical processes that took place at the surface of the asteroids (Sect. 3). The results of spectral analysis for each of the eight objects are described in Sect. 4. Some general characteristics of our sample are discussed in Sect. 5. Finally, the conclusions summarize the obtained results.

\section{The observing method and data reduction}

In contrast to the main-belt asteroids, the asteroids classified as NEA do not often have a favorable geometry for ground-based observations. The small diameters of the majority of NEAs impose tight constraints on the suitable geometries of observations for determining the reflective properties of their surfaces. These conditions are usually met in the case of a close approach to the Earth, when the apparent magnitude decreases by several magnitudes. These suitable geometries occur on average, only five times per century.

During the observing run, the asteroids and the solar ana$\log$ were alternatively observed. Our strategy was to observe all asteroids as close to the zenith as possible (Table 1). Each observed asteroid was preceded by observations of solar analogs located in the vicinity. The following stars were observed and used as solar analogs: BD+41 309, HD 154716, HD 217577 , HD 27834, BD+28 3198, HD 216516, and L115-271 (Table 1). Our choice was to observe the solar analogue as close as possible to the target. The differential airmass between the asteroid and the standard was usually restricted to less than 0.15 . The photometric G2V standards were chosen. We made an exception for 2010 TD54, where the data reduction were performed using L115-271, commonly used in NIR spectral measurements.

The asteroids were observed in the $0.8-2.5 \mu \mathrm{m}$ spectral region with the SpeX/IRTF instrument, located on Mauna Kea, Hawaii. These observations were performed remotely from the Centre d'Observation à Distance en Astronomie à Meudon (CODAM) (Birlan et al. 2004, 2006) using the low resolution prism mode $(R=100)$ of the spectrograph. We used a $0.8 \times 15$ arcsec slit oriented north-south. The spectra for the asteroid and the solar analog stars were obtained alternatively at two separate locations along the slit denoted A and B following the nodding procedure (Nedelcu 2010).

The data reduction process consists of two main steps (Birlan et al. 2007; Nedelcu 2010): first, obtaining the raw spectra for the object and the solar analog and second, computation of a 
Table 2. Some characteristics of our NEAs: orbit type, semi-major axis, eccentricity, inclination, absolute magnitude $(H)$, the Delta- $V$, and taxonomic classification.

\begin{tabular}{|c|c|c|c|c|c|c|c|c|}
\hline \multirow[t]{2}{*}{$\overline{\text { Object }}$} & \multirow[t]{2}{*}{ Orbit type } & \multirow[t]{2}{*}{$\bar{a}$} & \multirow[t]{2}{*}{$\bar{e}$} & \multirow[t]{2}{*}{$i$} & \multirow{2}{*}{$\begin{array}{c}\Delta V \\
{\left[\mathrm{~km} \mathrm{~s}^{-1}\right]}\end{array}$} & \multirow[t]{2}{*}{$\bar{H}$} & \multicolumn{2}{|c|}{ Taxonomic type } \\
\hline & & & & & & & Previous & This work \\
\hline (1917) Cuyo & Amor & 2.15005205 & 0.50448184 & 23.943786 & 8.556 & 14.7 & $\mathrm{Sl} ; \mathrm{S}$ & Srw \\
\hline (5620) Jasonwheeler & Amor & 2.15783969 & 0.42369152 & 7.861788 & 6.974 & 17.0 & - & $\mathrm{D} ; \mathrm{T}$ \\
\hline (8567) 1996 HW1 & Amor & 2.04580925 & 0.44905867 & 8.439303 & 6.495 & 15.4 & $\mathrm{~S}$ & $\mathrm{Sq}$ \\
\hline (16960) 1998 QS52 & Apollo & 2.20249841 & 0.85791440 & 17.563883 & 11.11 & 14.2 & $\mathrm{Sq} ; \mathrm{Q}$ & $\mathrm{Sr}$ \\
\hline (164400) 2005 GN59 & Apollo & 1.65644063 & 0.46770919 & 6.627004 & 6.002 & 17.4 & - & $\mathrm{L}$ \\
\hline (188452) 2004 HE62 & Amor & 2.55781560 & 0.56690184 & 24.685809 & 9.074 & 17.3 & - & $\mathrm{Sr} ; \mathrm{Sv}$ \\
\hline 2001 SG286 & Apollo & 1.35819973 & 0.34708703 & 7.772096 & 5.604 & 20.9 & D & $\mathrm{D}$ \\
\hline 2010 TD54 & Apollo & 1.97198039 & 0.64352131 & 4.809727 & - & 28.7 & $\mathrm{~S}$ & $\mathrm{Sr} ; \mathrm{Sv}$ \\
\hline
\end{tabular}

normalized reflectance spectrum by dividing the asteroid spectrum by the solar analog spectrum and performing a correction for telluric lines.

For the first step, the Image Reduction and Analysis Facility (Tody 1986) was used. Preprocessing of the CCD images included bias and flat field correction. An averaged bias frame taken at the telescope at the beginning of each observing night was used to perform bias subtraction. Flat fields images were obtained for each object using calibration lamps, at the beginning or end of the night. For the wavelength calibration, the Ar lamp spectrum was used. In the second step, specific IDL routines were used to help diminish the influence of telluric bands in our spectra (Rivkin et al. 2004). No other correction for the differential refraction was performed. For the computation of the final reflectance (ratio of the asteroid spectrum to the star spectrum), we took into account the similar dynamic regimes of the detector (Vacca et al. 2004; Rayner et al. 2003).

Log of asteroids observations is given in Table 1. In general, the asteroid spectra were obtained taking images with an integration time (Itime) of $120 \mathrm{~s}$ in the nodding procedure, for several cycles, to increase the $S / N$ ratio. For two objects of our sample (2005 GN59, and 2001 SG286), the atmospheric conditions and their low brightness imply a poor $S / N$ ratio. In this case, to obtain reliable spectral measurements, the images were selected by visual inspection, removing all those in which we could not distinguish the trace of the spectrum before the data reduction procedure.

\section{Methods used to analyze data}

We consider our analysis of spectra in the context of previously published physical and dynamical properties of these objects. Table 2 summarizes some parameters of our sample.

We complete our spectral data with the visible counterpart, when available. This is the case for four of our asteroids: (1917) Cuyo, (8567) 1996 HW1, (16960) 1998 QS52, and 2001 SG286. For each of them, the visible spectrum was merged with our NIR data using a procedure of minimization of data in the common spectral region $0.82-0.9 \mu \mathrm{m}$.

We computed the slope for each spectrum using a first-order polynomial fit. For the case of composite spectra $(V+\mathrm{NIR})$, the slope was computed for the spectra normalized to $0.55 \mu \mathrm{m}$ to compare with the conclusions of the DeMeo et al. (2009) taxonomy. Otherwise, when only NIR was available, the slope was computed for the spectra normalized to $1.25 \mu \mathrm{m}$.

Taxonomic types, although not usable to determine the mineralogic compositions of the objects, help constrain mineral species that may be present on the surface of the asteroid. Currently, the most commonly taxonomies are: Tholen taxonomy (Tholen 1984), Barucci taxonomy (Barucci et al. 1987; Birlan et al. 1996), based on Eight-Color Asteroid Survey data (Zellner et al. 1985), SMASII spectral taxonomy (Bus \& Binzel 2002), and Bus-DeMeo taxonomy (DeMeo et al. 2009). We used the last one, which is an extension of the Bus \& Binzel (2002) taxonomy to the near-infrared, considering the data spanning the wavelength range between $0.45 \mu \mathrm{m}$ to $2.45 \mu \mathrm{m}$. The Bus-DeMeo taxonomy is based on Principal Component Analysis and comprised 24 classes. This taxonomy allows us to analyze spectra using only NIR data, although we are in this case unable to obtain a unique classification. We used two independent methods to establish the taxonomical class of each asteroid in our sample. In a first approach, spectral data of our asteroids were compared with Bus-DeMeo taxonomic classes via the MIT-SMASS on-line tool ${ }^{2}$. The second approach to taxonomic classification was a procedure developed during this study using a $\chi^{2}$ minimization method accounting for the mean and standarddeviation values of the Bus-DeMeo taxonomic classes. For this method, we define a reliability criterion:

Reliability $=\frac{\operatorname{card}\left(\left[\lambda_{m}, \lambda_{M}\right] \cap\left\{\lambda_{1}^{\mathrm{T}}, \lambda_{2}^{\mathrm{T}}, \ldots, \lambda_{41}^{\mathrm{T}}\right\}\right)}{41}$

where $\left[\lambda_{m}, \lambda_{M}\right]$ is the spectral interval between the minimum and maximum wavelengths of the spectrum, $\lambda_{1}^{\mathrm{T}}, \lambda_{2}^{\mathrm{T}}, \ldots, \lambda_{41}$ are the 41 wavelengths from Bus-DeMeo taxonomy, spanning the interval between $0.45 \mu \mathrm{m}$ and $2.45 \mu \mathrm{m}$, and $\operatorname{card}()$ represents the number of elements of a discrete set. To apply this procedure, we smoothed our data by curve fitting with polynomial functions. This was done using poly fit from the Octave 3.2 computation environment. The degree of the polynomial was selected to be between 15 to 21 such that the fit produces the smallest least squares fitting residuals. The obtained fitting curves are given in Fig. 3. Overall, we observed that both procedures gave similar results.

We also compared our observational data with laboratory spectra. Spectroscopy of different samples made in the laboratory provides the basis upon which compositional information about unexplored or unsampled planetary surfaces is derived from remotely obtained reflectance spectra. The Relab ${ }^{3}$ spectral database contains more than 15000 spectra for different types of materials from meteorites to terrestrial rocks, man-made mixtures, and terrestrial and lunar soils. The comparison was made in the first step using a $\chi^{2}$ minimization method between asteroid spectrum and all the spectra from the Relab database, which were first normalized to $1.25 \mu \mathrm{m}$. In this way, the best fifty spectral curves of different Relab samples were selected for further analysis. From these results, only the meteorite spectra were

\footnotetext{
${ }^{2}$ http://smass.mit.edu/busdemeoclass.html

${ }^{3}$ http://www.planetary.brown.edu/relab/
} 
kept. After that, we considered only those meteorite spectra for which the mean reflectance value remains roughly in an interval of $\pm 50 \%$ centered on the albedo value (or mean albedo value corresponding to taxonomic class for that asteroid). A third selection was made by taking into account the spectral-feature position (band maxima, band minima) and slope. This was done by comparing each asteroid spectrum with a meteorite spectrum, and selecting the meteorite spectra for which the spectral features do not differ by roughly more than $10 \%$ and the difference in slope is not notably larger than $10 \%$. Our initial intention had been to publish the first three most closely fitting solutions satisfying this criteria, for each asteroid spectrum. However, for some asteroid spectra we found only one or two solutions corresponding to the above mentioned criteria.

In our sample, the asteroids belonging to the S-complex were also investigated by considering space weathering effects. Our approach involved applying the model proposed by Brunetto et al. (2006) and calculating the $C_{\mathrm{s}}$ parameter for each of these objects using the formula:

$W(\lambda)=K \times \exp \left(\frac{C_{\mathrm{s}}}{\lambda}\right)$

Brunetto et al. (2006) demonstrated that for laboratory experiments this provides a good approximation of the effects of irradiated materials, regardless of whether they are powder or bulk samples, meteorite or terrestrial samples, or samples of either olivine or orthopyroxene. They concluded that a weathered spectrum can be obtained by multiplying the spectrum of the unaltered sample by the exponential function (Eq. (2)) depending on the precise value of the parameter $C_{\mathrm{s}}$.

$C_{\mathrm{s}}=\alpha \times \ln (\beta \times d+1)$

where $\alpha=-0.33 \mu \mathrm{m}$ and $\beta=1.1 \times 10^{19} \mathrm{~cm}^{2}$.

Brunetto \& Strazzulla (2005) demonstrated that ion-induced spectral reddening is related to the formation of displacements, though this $C_{\text {s }}$ parameter is also correlated with the number of displacements per $\mathrm{cm}^{2}$ (damage parameter, noted here by the letter $d$ ). By fitting experimental data, Brunetto et al. (2006) obtained the relation between $C_{\mathrm{s}}$ and the number of displacements per $\mathrm{cm}^{2}$ (Eq. (3)). We computed this damage parameter by considering the values from Brunetto et al. (2006).

This model for the space weathering effects, which we applied to our data, describes the effects of solar-wind ion irradiation. This is not the only active weathering process, but it seems to be the most efficient at $1 \mathrm{AU}$ (Vernazza et al. 2009; Brunetto et al. 2006).

We managed to remove the effects of space weathering by dividing the spectrum with the computed exponential continuum $W(\lambda)$. The de-reddened spectra obtained for the S-type asteroids were compared again with the laboratory measurements from the Relab database.

The computations for the modeling methods described above were done using M4AST ${ }^{4}$ (Popescu \& Birlan 2011), which is software developed at IMCCE Paris to analyze asteroid spectra. This tool implements the algorithms for the aforementioned models.

$\frac{\mathrm{OPX}}{\mathrm{OPX}+\mathrm{OL}}=0.4187 \times\left(\frac{\mathrm{BII}}{\mathrm{BI}}+0.125\right)$.

Since for (1917) Cuyo, (8567) 1996 HW1, and 16960(1998 QS52) we had V+NIR spectra, we were able to apply the model

\footnotetext{
${ }^{4}$ http://cardamine.imcce. $\mathrm{fr} / \mathrm{m} 4$ ast/
}

proposed by Cloutis et al. (1986). Thus, we computed the twoband centers (at $1 \mu \mathrm{m}$ and $2 \mu \mathrm{m}$ ), the ratio of the areas of the second to the first absorption band (BAR) and we analyzed the percentage of orthopyroxene using Eq. (4). The computations were done using the standard procedures described by Cloutis et al. (1986). These results are presented in Table 5.

\section{Results}

This section describes the results obtained for the observed asteroids: (1917) Cuyo, (5620) Jasonwheeler, (8567) 1996 HW1, (16960) 1998 QS52, (188452) 2004 HE62, 2010 TD54, (164400) 2005 GN59, and 2001 SG286. All spectra were normalized to $1.25 \mu \mathrm{m}$. The spectra for the first six objects are plotted in Fig. 1 with error bars and joined with the visible part available from the literature (Binzel et al. 2004b; Vernazza 2006). The data obtained for the last two objects, (164400) 2005 GN59 and 2001 SG286, are plotted in Fig. 4 together with some curves that model these spectra.

The discussion about the taxonomic type of each object is made with reference to Fig. 3 . The results for the taxonomic classification of spectra are synthesized in Table 2 to allow a comparison with the physical properties and previously taxonomic classification.

Table 3 summarizes the comparison of asteroid spectra with those of meteorites from the Relab database considering both the original and de-reddened spectra (the case of S-type asteroids). The corresponding figures are presented in Appendices A and B. Some additional data related to meteorites with similar spectra to our objects are given in Table 3 .

\section{1. (1917) Cuyo}

With an absolute magnitude $H=14.7$, this object has an estimated diameter of $5.2 \mathrm{~km}$ (Binzel et al. 2002). It is an Amor-type asteroid,with a synodic period of $2.6905 \pm 0.0005 \mathrm{~h}$ (Wisniewski et al. 1997).

Two spectra in the visible are published for this object. For the first one, Binzel et al. (2004b) found that this asteroid is a Sl-type in Bus taxonomy, with a high slope of $\left(0.7233 \mu \mathrm{m}^{-1}\right)$. The second one was classified by Michelsen et al. (2006) as an S-type asteroid in Tholen taxonomy. We joined the visible spectrum from the SMASS database corresponding to Binzel et al. (2004b), with our data in NIR region (Fig. 1). The analysis was made on the composite $V+$ NIR spectrum.

With the tool from the MIT-SMASS website, this NEA was classified as Sr-type with a higher spectral slope of $0.5086 \mu \mathrm{m}^{-1}$. Using our $\chi^{2}$ method, R- and Sr-types are obtained as possible classes for this object. The R-type is obtained with a slightly better coefficient of reliability than Sr-type, because of the trend in the $1-1.5 \mu \mathrm{m}$ spectral region. By visual inspection of the two solutions, we can see that the features around $1 \mu \mathrm{m}$ and $2 \mu \mathrm{m}$ are more shallow than for R class (Fig. 3), so we can conclude that this object is an Sr type asteroid.

The comparison with the Relab database shows that the closest spectral fit is obtained for a tiny section from the Dhajala meteorite (Sample ID: LM-LAM-026, Fig. A.1). This corresponds to an ordinary chondrite meteorite rich in Fe (H3-4 olivinebronzite). Das Gupta et al. (1978) estimated a total iron content of $27.1 \%$ of the total mass of Dhajala. This meteorite was also studied by analyzing the metallic grains in its OC structure (Kong \& Ebihara 1997). While the formation of metallic iron is a consequence of the spatial alteration of an object, space weathering models are nevertheless justified. 

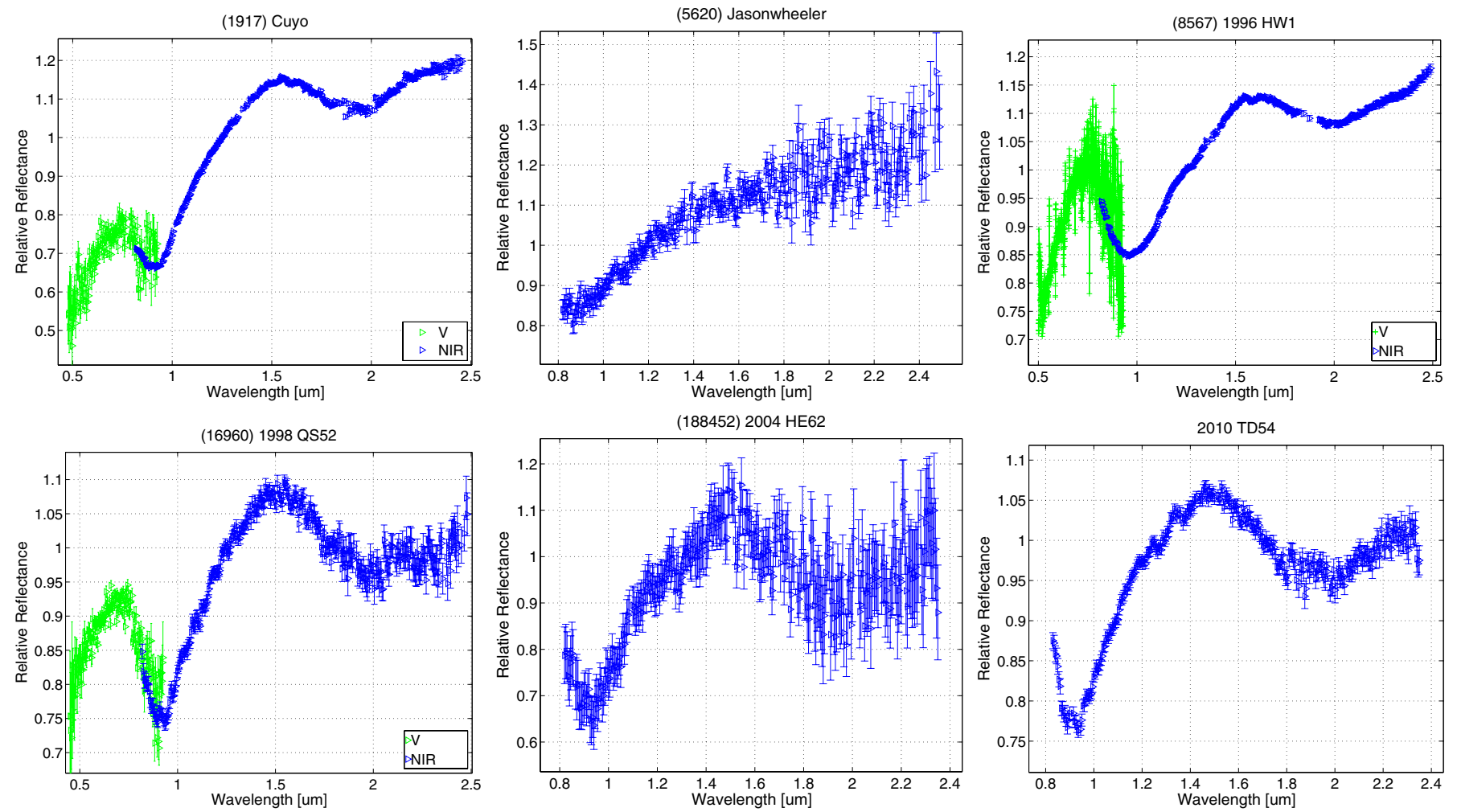

Fig. 1. Spectra of (1917) Cuyo, (5620) Jasonwheeler, (8567) 1996 HW1, (16960) 1998 QS52, (188452) 2004 HE62, and 2010 TD54, with errorbars. All spectra are normalized to $1.25 \mu \mathrm{m}$. For (1917) Cuyo, (8567) $1996 \mathrm{HW}$, and (16960) 1998 QS52, we added the visible part (plotted with green) from the literature (Binzel et al. 2004b; Vernazza 2006).

Modeling the effects of space weathering on the basis of the exponential continuum, we find that $C_{\mathrm{s}}=-0.484 \mu \mathrm{m}$, corresponding to strong spectral reddening. Owing to the size of this NEA, this value agrees with the general conclusion that larger objects are collisionally older, hence contain surfaces that are more space-weathered, or are not subject to other surface rejuvenating events as frequently as smaller NEAs. The number of displacements per $\mathrm{cm}^{2}$, which provides a measure of the solarwind ion irradiation, is $3.25 \times 10^{19}$, which implies that the exposure has been longer than 1My (Brunetto et al. 2006).

By removing the exponential continuum and fitting the unweathered spectrum with meteorite spectra from the Relab database, the closest match was found for ordinary chondrites with high level content of $\mathrm{Fe}$ but with a higher petrological type (H5, H6). The spectra of the following meteorites are very similar to the de-reddened spectrum of (1917) Cuyo: Lancon, Collescipoli, Ehole (Table 3, Fig. B.1).

\section{2. (5620) Jasonwheeler}

This object has the geometric albedo $p_{v}=0.094$ (Mueller et al. 2011). It is an Amor-type asteroid with a diameter of $1.77 \mathrm{~km}$ (Mueller et al. 2011) and the synodic period of $5.307 \pm 0.001 \mathrm{~h}$ (Durkee 2010). The light-curve amplitude of 1.2 magnitude (Durkee 2010) is indicative of an object with an elongated shape, or a binary system. Having $\Delta V=6.974 \mathrm{~km} \mathrm{~s}^{-1}$, this asteroid is a suitable target in terms of propulsion for a possible spacecraft mission.

No other spectroscopic studies of this object were found in the literature. The NIR spectrum obtained on May 5, 2009 when the object had the apparent magnitude 16.5, is plotted in Fig. 1.
The MIT-SMASS online tool for taxonomy classified this object as belonging to D-class. With our best-fit method, the spectrum is more similar to T-class (Fig. 3). In general, D-type asteroids have linear spectra with a very steep slope (greater than $0.38 \mu \mathrm{m}^{-1}$ ) and display a slight curvature around $1.5 \mu \mathrm{m}$. On the other hand, T-types also have linear spectra with a steep gradient - between 0.25 and $0.38 \mu \mathrm{m}^{-1}$ - that nevertheless gradually curves concavely downward (DeMeo et al. 2009). The spectrum of this asteroid has a steep slope in the $0.9-1.5 \mu \mathrm{m}$ region and a slight curvature between 1.5-2.2 $\mu \mathrm{m}$ (Fig. 3), though the classification is at the boundary between D-type and T-type. The overall NIR slope is $0.2504 \mu \mathrm{m}^{-1}$.

Taking into account the low geometrical albedo when comparing with spectra from the Relab database, we found close spectral matches for this spectrum with CM2 carbonaceous chondrite meteorites (Table 3, Fig. A.2). In general, the CM2 meteorites are characterized by $30 \%$ levels of chondrules with grain sizes of $\approx 300 \mu \mathrm{m}$, the absence of $\mathrm{Fe}-\mathrm{Ni}$ alloys, and the presence of CAI (Ca-Al inclusions) (Dobrica 2010). The closest description of the spectrum is provided by a sample of particulates $(0-75 \mu \mathrm{m})$ from the meteorite Mighei/Meghei (Sample ID: MR-MJG-108). Other spectra of CM2 carbonaceous chondrite meteorites that fit the Jasonwheeler NIR spectrum are those of powdered samples with particle sizes smaller than $125 \mu \mathrm{m}$. This fit suggests that the asteroid might be covered by a fine regolith layer.

By fitting the spectrum (Fig. 2) with an eighth order polynomial function, we can observe an excess of flux after $2.2 \mu \mathrm{m}$ that cannot be explained by the general trend in the spectral region 1.4-2.2 $\mu \mathrm{m}$ and its taxonomical classification. Even if the level of noise is relatively important, it can be assumed that this 
Table 3. Summary of results obtained by matching the asteroid spectra and de-reddened asteroid spectra with spectra from the Relab database.

\begin{tabular}{|c|c|c|c|c|c|}
\hline & \multicolumn{5}{|c|}{ Matching results for asteroid spectra } \\
\hline \multirow{5}{*}{$\begin{array}{l}\text { Spectrum } \\
\text { (1917) Cuyo } \\
\text { (5620) Jasonwheeler }\end{array}$} & Meteorite & Sample ID & Type & Texture & Size $[\mu \mathrm{m}]$ \\
\hline & Dhajala & LM-LAM-026 & $\mathrm{OC} / \mathrm{H} 3-4$ & Thin Section & - \\
\hline & Meghei (Mighei) & MR-MJG-108 & $\mathrm{CC} / \mathrm{CM} 2$ & Particulates & $\overline{0-75}$ \\
\hline & Cold Bokkeveld & MB-ТXH-061 & $\mathrm{CC} / \mathrm{CM} 2$ & Particulates & $0-125$ \\
\hline & ALH84029 & MB-TXH-052 & $\mathrm{CC} / \mathrm{CM} 2$ & Particulates & $0-100$ \\
\hline (8567) $1996 \mathrm{HW1}$ & Hamlet & OC-TXH-002-C & OC/ LL4 & Particulates & $0-125$ \\
\hline \multirow[t]{3}{*}{ (16960) 1998 QS52 } & Saratov & MB-CMP-028-H & OC/L4 & Particulates & $0-370$ \\
\hline & Homestead & MR-MJG-048 & OC/L5 & - & - \\
\hline & Hamlet 1 & MR-MJG-069 & OC/LL4 & - & - \\
\hline \multirow[t]{3}{*}{ (188452) 2004 HE62 } & La Criolla & MH-FPF-050-B & OC/L6 & Particulates & $0-150$ \\
\hline & Cherokee Springs & OC-TXH-001-A & OC/LL6 & Chip & - \\
\hline & Wold Cottage & MH-FPF-064 & OC/L6 & Particulates & - \\
\hline \multirow[t]{4}{*}{2010 TD54 } & Saratov & MB-CMP-028-B & OC/L4 & Particulates & $10-45$ \\
\hline & Mirzapur & TB-TJM-111 & OC/L5 & Particulates & $0-150$ \\
\hline & Rio Negro & TB-TJM-081 & OC/L4 & Particulates & $0-150$ \\
\hline & \multicolumn{5}{|c|}{ Matching results for de-reddened asteroid spectra } \\
\hline \multirow[t]{3}{*}{ (1917) Cuyo } & Lancon & MR-MJG-033 & $\mathrm{OC} / \mathrm{H} 6$ & - & $\overline{-}$ \\
\hline & Collescipoli & MR-MJG-030 & $\mathrm{OC} / \mathrm{H} 5$ & - & - \\
\hline & Ehole & TB-TJM-074 & $\mathrm{OC} / \mathrm{H} 5$ & Particulates & $0-150$ \\
\hline \multirow[t]{3}{*}{ (8567) 1996 HW1 } & Cherokee Springs & TB-TJM-090 & OC/LL6 & Particulates & $0-150$ \\
\hline & Hedjaz & OC-TXH-016-C & OC/L3-6 & Particulates & $0-125$ \\
\hline & Ensisheim & TB-TJM-092 & OC/LL6 & Particulates & $0-150$ \\
\hline \multirow[t]{2}{*}{ (16960) 1998 QS52 } & Hamlet 1 & MR-MJG-069 & OC/LL4 & - & - \\
\hline & Gruneberg & MR-MJG-040 & $\mathrm{OC} / \mathrm{H} 4$ & - & - \\
\hline \multirow[t]{3}{*}{ (188452) 2004 HE62 } & Nanjemoy & MR-MJG-034 & $\mathrm{OC} / \mathrm{H} 6$ & - & - \\
\hline & Olmedilla de Alarcon & MR-MJG-075 & $\mathrm{OC} / \mathrm{H} 5$ & - & - \\
\hline & МАC88119.9 & MB-ТXH-044 & $\mathrm{OC} / \mathrm{H} 5$ & Slab & 0 \\
\hline \multirow[t]{3}{*}{2010 TD54 } & Gruneberg & MR-MJG-040 & $\mathrm{OC} / \mathrm{H} 4$ & - & - \\
\hline & Queen's Mercy & MR-MJG-035 & $\mathrm{OC} / \mathrm{H} 6$ & - & - \\
\hline & Ochansk & MR-MJG-027 & $\mathrm{OC} / \mathrm{H} 4$ & - & - \\
\hline
\end{tabular}

Notes. The comparison was made using a $\chi^{2}$ method and a selection of the obtained results was done based on spectral features (band, band-gap, concavity) positions, and albedo values. For (5620) Jasonwheeler, a de-reddening model was not applied. The figures for this comparison can be found in Appendices A and B.

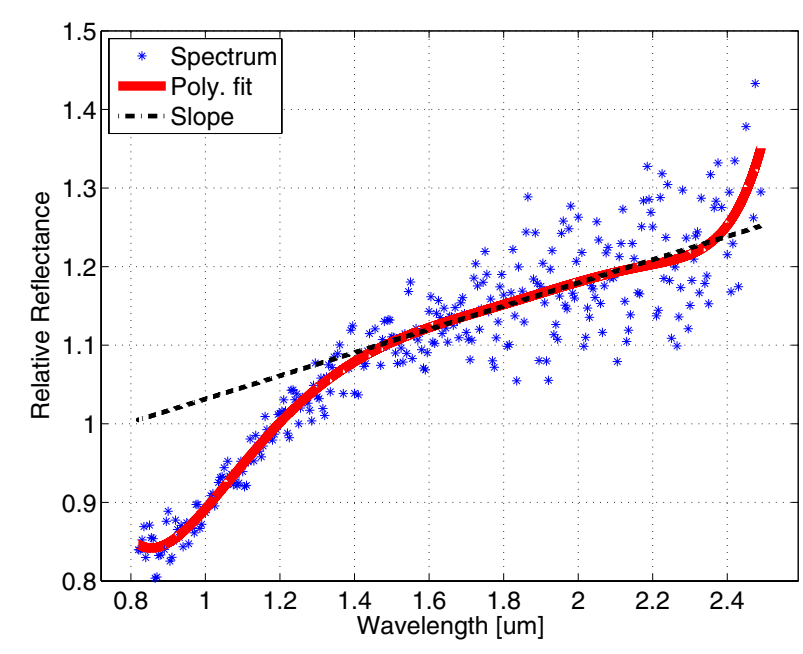

Fig. 2. Spectrum of (5620) Jasonwheeler. The dashed line indicates where a linearly extrapolated continuum would fall, the solid line shows the presence of thermal flux.

feature is caused by asteroid thermal emission. Following Rivkin et al. (2005), we calculated the "thermal excess" parameter that describes this phenomenon:

$\gamma=\frac{R_{2.5}+T_{2.5}}{R_{2.5}}-1=0.092 \pm 0.0420$ where $R_{2.5}$ is the reflected flux at $2.5 \mu \mathrm{m}$ and $T_{2.5}$ is the thermal flux at $2.5 \mu \mathrm{m}$. This value agrees with the geometrical albedo $p_{v}=0.094$ for an asteroid at a $1.345 \mathrm{AU}$ distance from the Sun and a phase angle of $20^{\circ}$ (Rivkin et al. 2005). This value also agrees with the result obtained from mid-IR observations by Mueller et al. (2011).

Taking into account its dynamical parameters and that $\mathrm{D}$ and T types are considered to be of a primitive composition, we can conclude that this object is very interesting from the point of view of "in situ" exploration.

\section{3. (8567) 1996 HW1}

This asteroid has an Amor type orbit and a $\Delta V=6.495 \mathrm{~km} \mathrm{~s}^{-1}$, though it is a suitable target in terms of propulsion for a space mission. The radar observations show a two-lobed object about 1.1 by $2.7 \mathrm{~km}$ in size (Taylor et al. 2009). The object is rotating with a synodic period of $8.7573 \pm 0.0009 \mathrm{~h}$ (Higgins et al. 2006).

Vernazza (2006) found this asteroid to be an S-type based on the visible spectrum $(0.5-0.95 \mu \mathrm{m})$ acquired on August 29, 2005 at TNG. Our NIR spectrum of (8567) 1996 HW1 was obtained in August 28, 2008 using an integration time of $60 \mathrm{~s}$, since the apparent magnitude was 12.9. We combined the visible spectrum from Vernazza (2006) with our NIR data (Fig. 1) before analyzing the composite spectrum.

Using the classification tool from the MIT-SMASS website, this NEA was classified as an S-type with the spectral slope 

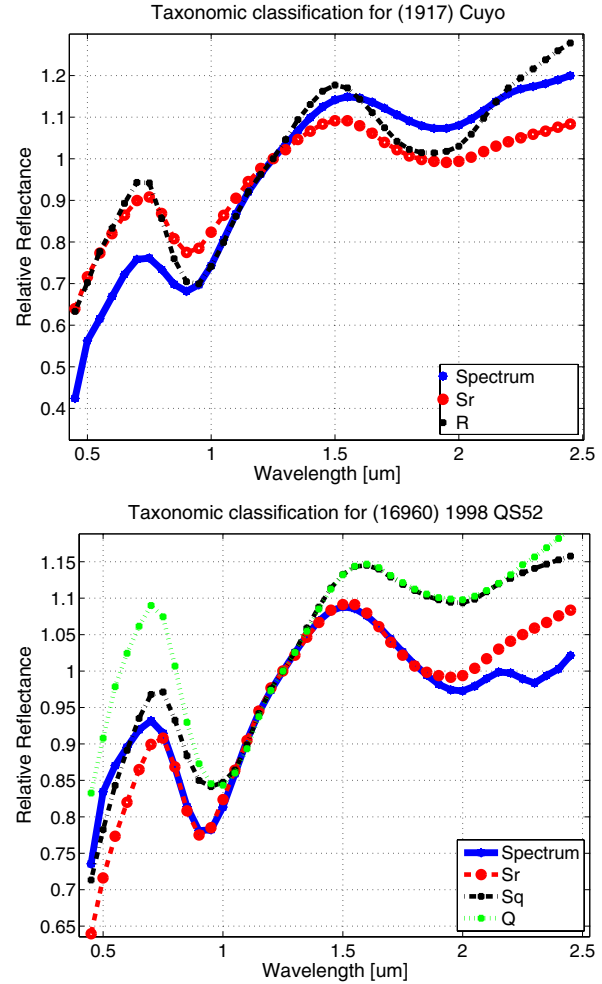

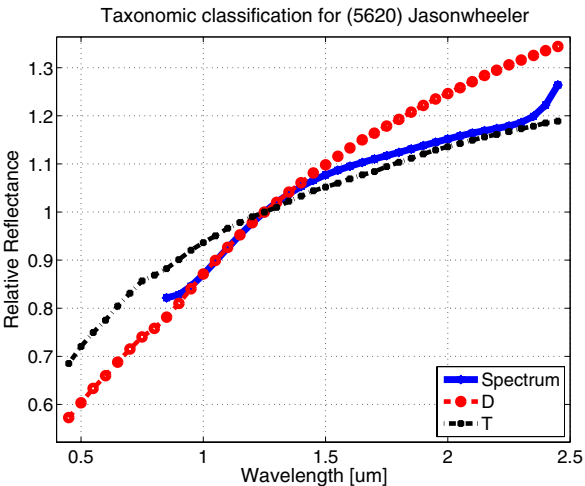

Taxonomic classification for (188452) 2004 HE62

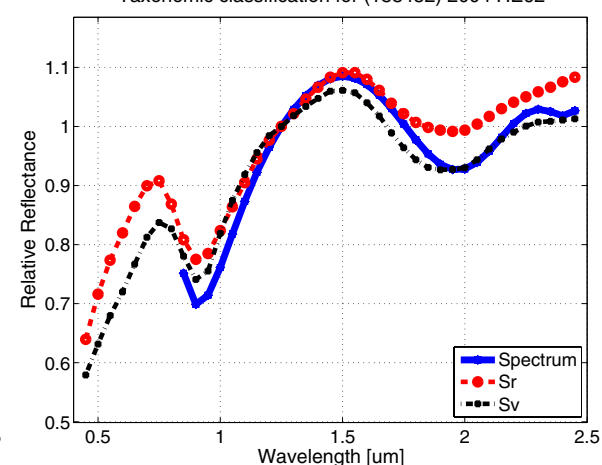

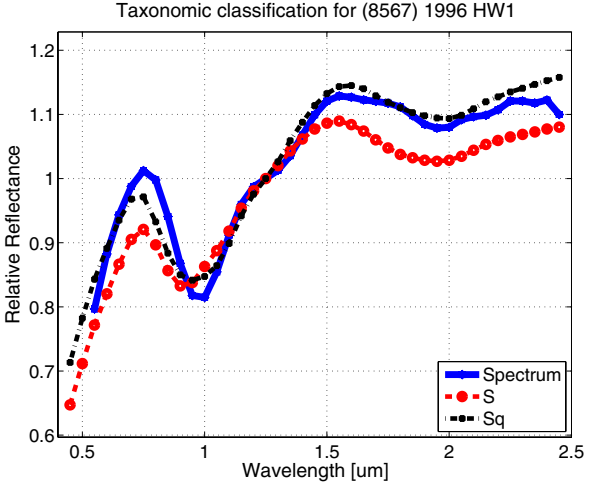

Taxonomic classification for 2010 TD54

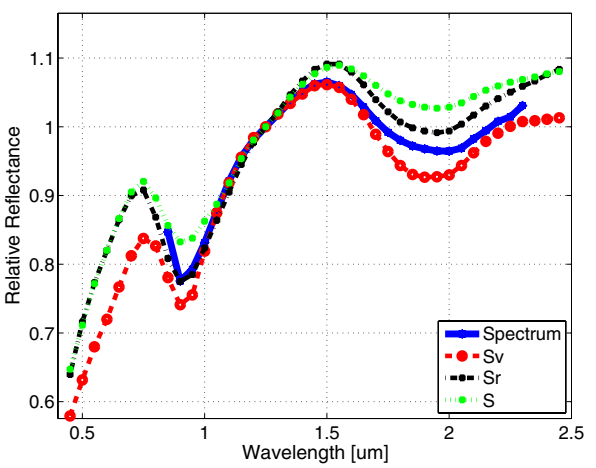

Fig. 3. Classification in Bus-DeMeo taxonomical system for (1917) Cuyo, (5620) Jasonwheeler, (8567) 1996 HW1, (16960) 1998 QS52, (188452) 2004 HE62, and 2010 TD54. The polynomial fit of the spectra are plotted against the curves for the resulting classes. The reflectances are normalized at $1.25 \mu \mathrm{m}$.

$0.2245 \mu \mathrm{m}^{-1}$. Using the $\chi^{2}$ approach, it can be found that an Sq type provides a closer fit spectrum than an S (Fig. 3), being at the transition between $\mathrm{S}$ and Q classes with two absorption bands around $1 \mu \mathrm{m}$ and $2 \mu \mathrm{m}$ that are more shallow than for Q-type (DeMeo et al. 2009). Comparing the features for the two considered spectral types with a polynomial fit of the spectrum, it can be observed that an Sq type matches more closely our data than an $\mathrm{S}$ type. Since there has been no measurement of the albedo for this object, assumes a value of 0.20 as typically found for the albedo of S-type asteroids (Fulchignoni et al. 2000), hence the diameter can be estimated to be $2.5 \mathrm{~km}$.

The spectrum from the Relab sample that provides the closest fit is a particulate ground sorted $(0-125 \mu \mathrm{m})$ Hamlet meteorite (sample ID: OC-TXH-002-C), an ordinary chondrite with a low level content of Fe and metal - LL4 (Table 3, Fig. A.3). The asteroid spectrum in the region $1.6-2.5 \mu \mathrm{m}$ is shallower than that of the meteorite spectrum, which could be explained by considering space-weathering effects.

Using the space weathering model of Brunetto et al. (2006), we calculated $C_{\mathrm{s}}=-0.258 \mu \mathrm{m}$, which corresponds to the reddening of the spectra. Modelling this with a damage parameter due to the solar-wind ion irradiation we found $d=1.08 \times$ $10^{19}$ displacements per $\mathrm{cm}^{2}$, thus it has experienced an appreciable amount of space weathering.

By removing the exponential continuum and comparing again with Relab meteorite spectra, we also found the closest match with an ordinary chondrite(LL6, L3-6 types) spectrum with a low metal, low Fe content. The meteorite spectra that provide the closest descriptions of the de-reddened spectrum of this asteroid are those of: Cherokee Spring, Hedjaz, and Ensisheim (Table 3, Fig. B.2). The Relab samples of these meteorites are particulates sorted in order of their sizes, which are smaller than $150 \mu \mathrm{m}$.

\section{4. (16960) 1998 QS52}

With an absolute magnitude $H=14.20$, this asteroid has an estimated diameter of $4.3 \mathrm{~km}$ (Binzel et al. 2002). It is characterized by a synodic period of $2.900 \pm 0.001 \mathrm{~h}$ (Warner 2009). It has an Apollo orbit type and $\Delta V=6.5 \mathrm{~km} \mathrm{~s}^{-1}$, which makes it an accessible target for a spacecraft mission. (16960) 1998 QS52 is a PHA object type with 0.01408 AU MOID computed at epoch 55600.0 MJD (Neodys ${ }^{5}$ ).

On the basis of a visible spectrum acquired with the MDM $2.4 \mathrm{~m}$ telescope in 15 October 1998, Binzel et al. (2004b) classified this asteroid as an Sq type. The spectrum of the visible region has a small negative slope of $-0.0205 \mu \mathrm{m}^{-1}$. We joined the SMASS visible spectrum with our NIR spectrum (Fig. 1). The following analysis was made on the combined $V+$ NIR spectrum.

Both methods of classification gave the same results: this object has the characteristics of an Sr type in Bus-DeMeo taxonomy, with a fairly $1 \mu \mathrm{m}$ feature (Fig. 3). The slope of this composite spectrum is $0.1126 \mu \mathrm{m}^{-1}$.

Comparison with meteorite spectra from Relab database shows the match with ordinary chondrites samples with low content of Fe (L4, LL4, L5). The best fit is a powdered sample (dimensions: $10-45 \mu \mathrm{m}$ ) from Saratov meteorite, an ordinary chondrite L4 (Table 3, Fig. A.4).

Analyzing this composite spectrum with a space weathering model (Brunetto et al. 2006), we computed the value of $C_{\mathrm{s}}=$ -0.149 which describes an unreddened spectrum corresponding to a fresh surface. It can be speculated that this young surface is due to a relatively recent close encounter with a planet (Binzel et al. 2010).

\footnotetext{
5 http://newton.dm.unipi.it/neodys/
} 
To verify this hypothesis, we generated 100 orbital clones of 1998 QS52 using a random Gaussian distribution centered at the nominal values in each of the six orbital elements. The $1 \sigma$ values were obtained from the orbital elements uncertainties provided by the Neodys service for this asteroid. The 100 clones were numerically integrated backward in time for 5000 years using the computing routines proposed by Nedelcu (2010). Each close encounter (MOID) with Venus, the Earth, and Mars was then carefully analyzed to find the closest one able to rejuvenate the surface of the object.

In addition to the 1989 close approach with Earth, an event already identified by Neodys, we were able to confirm that another five close approaches with Venus had occurred in the past 3000 years before the common origin signature of our cloud of clones was erased by close planetary encounters. The MOID values are larger than those predicted by Binzel et al. (2010), whose findings, however we cannot exclude. Our deterministic clones approach can reliably obtain NEAs positions only for a couple of thousands years backward in time (Nedelcu 2010). Systematic errors in osculating elements can affect the position of the object, and close approaches with telluric planets will modify in a nonlinear way the uncertainty in the position. Thus, we estimate that for timescales of millions of years (the scale for SW determined by Brunetto et al. 2006) the object might experience additional close encounters that cannot be reproduced by our numerical integration of orbit.

Comparing the de-reddened spectrum with the Relab database, we found a good fit to the spectrum with a Hamlet meteorite, a LL4 ordinary chondrite (Fig. A.4). This result was also found when comparing with the original spectrum. This agrees with our finding that dividing the spectrum with the exponential continuum by the small value of $C_{\mathrm{s}}$ does not alter its characteristics.

\section{5. (188452) 2004 HE62}

Few physical parameters of (188452) 2004 HE62 are known. This asteroid has an Amor orbit and an absolute magnitude $H=17.30$ (Table 2). No other spectral investigations have been published for this asteroid. Our NIR spectrum observations (Fig. 1) of this object were made on August 27, 2008 when the object had an apparent magnitude of 16.7 .

The spectrum of (188452) 2004 HE62 has two features around 1 and $2 \mu \mathrm{m}$ : these are two deep absorption bands that are larger than for Sv-type meteorites but not so deep to be classified as one of the end members R or V. However, the classification is between the $\mathrm{Sr}$ and $\mathrm{Sv}$ classes in the Bus-DeMeo taxonomy (Fig. 3). A visible spectrum would help us to clarify the object's classification, which in the end was obtained with both methods of classification. The spectral slope computed on the NIR part of the spectrum is $0.1167 \mu \mathrm{m}^{-1}$. Assuming an average albedo of 0.2 , which is typical of S-type objects, we can estimate the diameter to be $\sim 1 \mathrm{~km}$.

By comparing with data from the Relab database, this spectrum was found to be closely matched by the spectra of ordinary chondrite meteorites with low Fe, low metallic content, and high petrologic class (L6, L5, LL6) - see Table 3 and Fig. A.5 for details. The best-fit solution was obtained with a spectrum of a particulate sample $(0-150 \mu \mathrm{m})$ from the La Criolla meteorite (Sample ID: MH-FPF-050-B).

Modeling the spectra with the exponential continuum (Brunetto et al. 2006), the parameter $C_{\mathrm{s}}$ is found to be of $-0.377 \mu \mathrm{m}$, which characterizes a surface affected by space weathering effects. Removing this continuum and comparing with Relab meteorite spectra, the best fit is also an ordinary chondrite (Fig. B.4), the same petrologic class but with a high content of $\mathrm{Fe}$ (OC types H5, H6). The closest match in this case is a sample from a Nanjemoy meteorite, a H6 olivine-bronzite OC, which consists of $18 \%$ Fayalitic material (Fig. B.4).

\subsection{TD54}

The analysis of this object is interesting from the point of view of its size and the phenomena that occur on the surface of small bodies during a close encounter with Earth. With an absolute magnitude $H=28.75,2010$ TD54 was discovered by the Catalina Sky Survey in October 09, 2010. Having an Apollo orbit type, this object passed within $0.00035 \mathrm{AU}$ of the Earth on 12.55 Oct. 2010 (Hicks \& Rhoades 2010).

Preliminary measurements were done by Hicks \& Rhoades (2010). They found a rotational period of $42.0 \mathrm{~s}$, which implies that this small NEA is the most rapidly rotating natural body known in the solar system. They also measured the object's average colors $(B-R=1.284 \pm 0.045 \mathrm{mag} ; V-R=$ $0.461 \pm 0.030 \mathrm{mag} ; R-I=0.344 \pm 0.022 \mathrm{mag})$. These are compatible with an S-type spectral classification.

The NIR spectrum of 2010 TD54 is plotted in Fig. 1. Using the MIT-SMASS online tool for Bus-DeMeo taxonomy, this asteroid is classified as belonging to a $\mathrm{S}$ complex, of subtypes $\mathrm{Sr}$ or Sq. An end class Q is also proposed but with a lower coefficient. By using the $\chi^{2}$ method, this spectrum can be classified to be between $\mathrm{Sv}$ and $\mathrm{Sr}$ classes (Fig. 3). It has a fairly prominent feature around $1 \mu \mathrm{m}$ and another around $2 \mu \mathrm{m}$. When considering these two results and the depth of the two absorption bands, we found that the Sr type provides a more accurate description for this object. The slope for this NIR spectrum is $0.062 \mu \mathrm{m}^{-1}$.

The matching with meteorite spectra (Fig. A.6) shows that the best fit is a spectrum for a sample from Saratov - an ordinary chondrite meteorite with a low content of Fe (L4). This sample contains particles with sizes between 10 and $45 \mu \mathrm{m}$ (Sample ID: MB-CMP-028-B). The spectrum can also be closely fitted with spectra of powdered samples from the meteorites Mirzapur and Rio Negro, which are also L ordinary chondrites.

Modelling the space weathering effects, we computed $C_{\mathrm{s}}=$ $-0.223 \mu \mathrm{m}$, which describes a relatively fresh surface. This agrees with the small bodies having relatively young surfaces, and Earth encounters being one of the origins for rejuvenating surfaces on near-Earth asteroids (Binzel et al. 2010). Removing the exponential continuum and comparing again with spectra from the Relab database, we found a good fit to the spectrum with those of ordinary chondrite meteorites with high level of Fe, from petrologic class 4 (H4 - olivine-bronzite). Spectra of meteorites such as Gruneberg, Queen's Mercy, or Ochansk match the unweathered spectrum of this asteroid (Table 3, Fig. A.6).

\section{7. (164400) 2005 GN59}

This asteroid has an absolute magnitude $H=17.40$, derived from astrometric observations. The synodic period of the asteroid was estimated to be $38.62 \pm 0.01 \mathrm{~h}$ (Vander Haagen 2011), but the monomodal solution of $19.31 \pm 0.01 \mathrm{~h}$ cannot be totally excluded.

A preliminary spectrum of this object was presented by Birlan et al. (2009), while Taylor et al. (2009) presented thermal emission data corroborated with radar observations. From these radar observations, Taylor et al. (2009) uncovered that this 

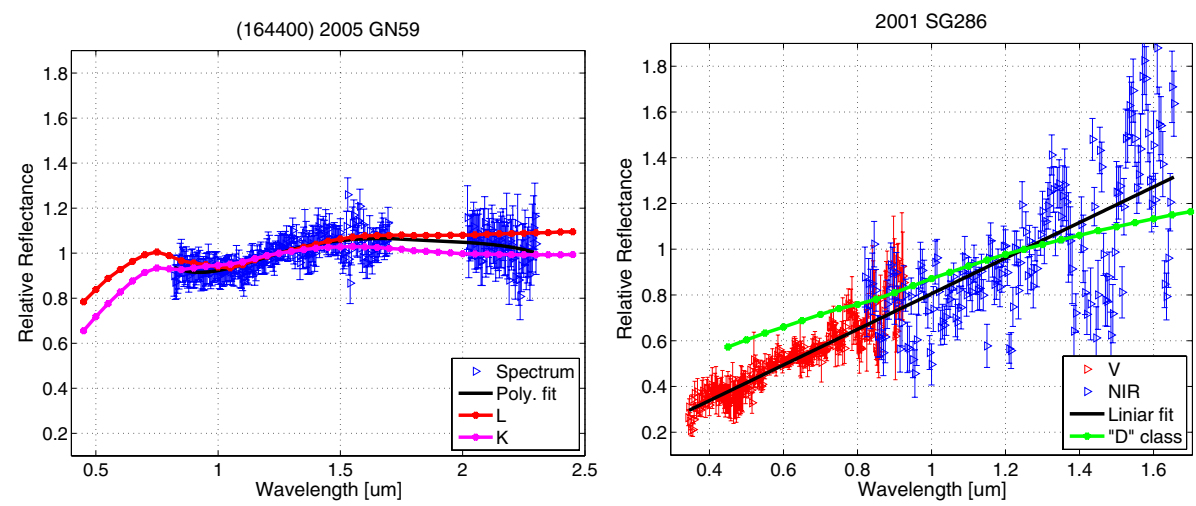

Fig. 4. NIR spectrum of (164400) 2005 GN59 and 2001 SG286. These spectra are normalized to $1.25 \mu \mathrm{m}$.

object has a two-lobed 0.35 by $1.1 \mathrm{~km}$ shape, with non-convex surface features.

Dynamically, (164400) 2005 GN59 is an Apollo asteroid. Its calculated $\Delta V=6.002 \mathrm{~km} \mathrm{~s}^{-1}$ imply that it is a suitable target in terms of propulsion for spacecraft mission.

The NIR spectrum of 164400 was obtained in August 28, 2008 for a total integration time of $480 \mathrm{~s}$. While the spectrum is quite noisy, to obtain information about its taxonomic class, we used a five order polynomial function to reproduce the real data. The values for reflectance corresponding to wavelengths between 1.7 and $2 \mu \mathrm{m}$ were excluded because of the very high noise caused by atmospheric turbulence (Fig. 4).

Both the MIT-SMASS on-line tool and the $\chi^{2}$ routine classify this object as an L-type. However, the $\mathrm{K}$ taxonomic class is also a reasonable match to our data (Fig. 4).

An additional NIR spectrum of this object was obtained by the MIT-UH-IRTF Joint Campaign for NEO Spectral Reconnaissance $^{6}$. This spectrum has higher $\mathrm{S} / \mathrm{N}$ than ours. We classified this spectrum with both methods and found it to be between $\mathrm{Sq}$ and Q types, while a K taxonomic class was proposed as a third solution. The $\mathrm{L}$ taxonomic class is also considered as a possible solution by the MIT-SMASS on-line tool. The difference between our spectrum and this one is caused by the low signal-to-noise ratio of our spectrum, which prevented us taking into consideration the feature between 1.7 and $2 \mu \mathrm{m}$. A visible spectrum would again help us to distinguish between the five possible solutions for the NIR part of the spectrum.

The spectrum of 2005 GN59 is noisy and we did not attempt to compare it with the Relab database and the de-reddening model.

\subsection{SG286}

This is an Apollo type asteroid with an absolute magnitude of 20.9. It is classified as PHA. Its $\Delta V=5 \mathrm{~km} \mathrm{~s}^{-1}$ makes it a suitable target for a spacecraft mission. Michel \& Delbo (2010) estimated its median lifetime as an NEA to be about 22.19 Myr. The mechanism of injection into the NEA population is the secular $v_{6}$ resonance, but the 3:1 mean motion resonance with Jupiter could not be entirely excluded (Michel \& Delbo 2010).

On the basis of spectral data in the visible region, Binzel et al. (2004a) classified this asteroid as a D-type one. Using an average albedo of 0.09 for D-type asteroids, Binzel et al. (2004a) computed a diameter of about $350 \mathrm{~m}$ for this object.

The object was observed on May 19, 2009 in the NIR for a total time of $480 \mathrm{~s}$, in difficult conditions (considerable

\footnotetext{
$\overline{{ }^{6} \text { http://smass.mit.edu/minus.html }}$
}

Table 4. Slope and $C_{\mathrm{s}}$ parameter for the S-type objects studied in this article.

\begin{tabular}{lcc}
\hline \hline Object & Slope $\left(\mu \mathrm{m}^{-1}\right)$ & $C_{\mathrm{s}}(\mu \mathrm{m})$ \\
\hline (1917) Cuyo & 0.5086 & -0.484 \\
(8567) 1996 HW1 & 0.2245 & -0.258 \\
$(16960)$ 1998 QS52 & 0.1126 & -0.149 \\
$(188452)$ 2004 HE62 $(*)$ & 0.1167 & -0.377 \\
2010 TD54 $(*)$ & 0.0620 & -0.223 \\
\hline
\end{tabular}

Notes. The calculation was made by normalization of spectra to $0.55 \mu \mathrm{m}$. Objects marked with $(*)$ are normalized to $1.25 \mu \mathrm{m}$ (only for NIR part).

differential motion, only a few hours of visibility over three nights, limited atmospheric transparency). The NIR spectrum is reliable only for the spectral interval $0.8-1.7 \mu \mathrm{m}$.

The composite V+NIR spectrum was obtained by superposing data in the $0.82-0.9 \mu \mathrm{m}$ spectral interval (Fig. 4). The slope parameter for the composite spectrum is $0.7202 \mu \mathrm{m}^{-1}$ (computed for a spectrum normalized to a reflectance value at $1.25 \mu \mathrm{m}$ ) in agreement with the slope range for D-type taxonomic class.

\section{Discussion}

Luu \& Jewitt (1990) suggested that the phase angle can affect the spectral slope. This was called "phase reddening" and consists of an increase in the spectral slope (reddening of the spectra) with the phase angle. Some studies have been performed based on laboratory measurements (Gradie \& Veverka 1986) and during the approach to (433) Eros by the NEAR spacecraft (Veverka et al. 2000). However, for our method of observation we retain the result mentioned in Binzel et al. (2004b) regarding a study conducted at MIT for which no correlation was found between the phase angle and the spectral slope for the ground-based asteroid reflectance spectra.

During our observing runs, all the asteroids were observed at phase angles as small as possible. Owing to this constraint, we succeeded in observing only six objects at a phase angles between $17^{\circ}$ and $30^{\circ}$ (Table 1). We observed (1917) Cuyo and (188452) $2004 \mathrm{HE62}$ at a phase angle around 60 (Table 1).

Assuming similar surface mineralogies, the influence of phase angle on spectral slope is unclear from our measurements. For (1917) Cuyo, a high spectral slope was obtained, but for (188452) 2004 HE62 the computed spectral slope is comparable to the spectral slope of (8567) $1996 \mathrm{HW} 1$ and (16960) 1998 QS52, which were measured at phase angles smaller than 

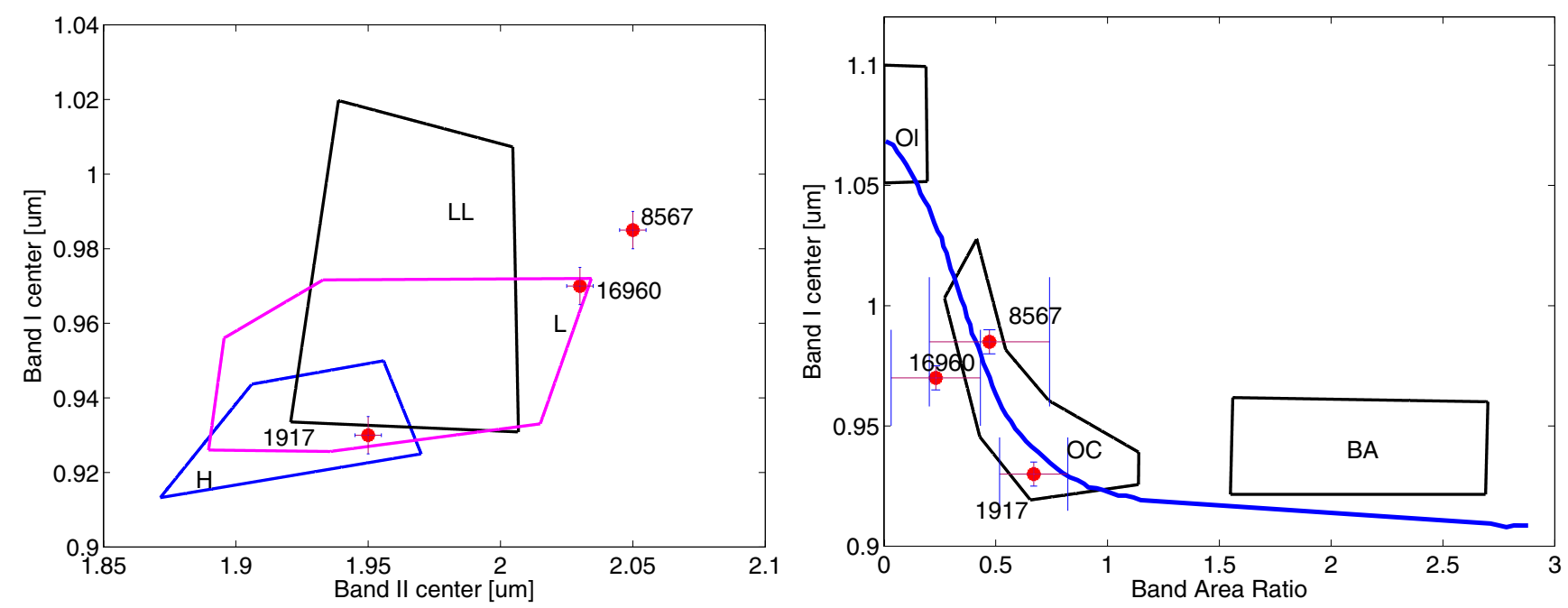

Fig. 5. (Left) Wavelength position of the centers of the two absorption bands computed using Cloutis et al. (1986). The regions enclosed correspond to the band centers computed for the H, L, and LL chondrites, respectively (de León et al. 2010). (Right) Band area ratio (BAR) versus band I centers. The regions enclosed by continuous lines correspond to the values computed for basaltic achondrites, ordinary chondrites (OC), and olivine-rich meteorites (Ol) (Gaffey et al. 1993).

$30^{\circ}$ (Table 4). Considering the trend of S-class objects, the reflectance value at $1.25 \mu \mathrm{m}$ is higher than the reflectance value at $0.55 \mu \mathrm{m}$, thus our comparison of slopes is correct. Therefore, no correction has been applied for this presumed effect of phase reddening.

Several NIR spectra for the asteroids (1917) Cuyo, (8567) 1996HW1, and (16960) 1998 QS52 were obtained by the MITUH-IRTF Joint Campaign for NEO Spectral Reconnaissance. These spectra are similar to those presented in this paper in terms of spectral features. However, a variation in spectral slope between the spectra of the same object was observed. Similar spectral variations in the NIR spectrum of a NEO was previously reported by de León et al. (2011). For our sample, the asteroid (8567) 1996HW1 was observed five times, at phase angles between $20^{\circ}$ and $55^{\circ}$. In this case, a variation in the spectral slope with phase angle was observed (i.e. the spectrum is redder for larger phase angles). While this object is not well-known in terms of spin axis and shape, it is difficult to draw any conclusions about the first order dependence of the slope on phase angle, while a surface dichotomy and degrees of space weathering could compete with this effect.

The S-types objects in our sample have widely varying spectral slopes (Table 4), which is a general conclusion for the asteroids belonging to this complex (DeMeo et al. 2009). In the Bus-DeMeo taxonomy, the objects in the S-complex with a slope larger than $0.25 \mu \mathrm{m}^{-1}$ receive the notation "w" added to their type as an indication that they may be affected by space weathering effects. This is the case for (1917) Cuyo.

Although space weathering may occur on all asteroids, many types lack strong spectral-band contrasts that ensure that weathering effects are easily detectable (Clark et al. 2002). S-class asteroids are significantly reddened compared with their presumed meteorite analog, and this difference can be explained by space weathering phenomenon (Vernazza et al. 2008). This process may be the result of dust impacts and solar wind sputtering on the surface of atmosphereless bodies and cause a reddening of the spectral slope, a decrease in spectral absorption intensities, and a diminishing of albedo (Fornasier et al. 2003).

An important concept in understanding space weathering processes is the development and accumulation of
Table 5. Computed parameters from the Cloutis et al. (1986) model applied to the V+NIR spectra of (1917) Cuyo, (8567) 1996 HW1, and (16960) 1998 QS52.

\begin{tabular}{lcccc}
\hline \hline Object & $\begin{array}{c}\text { BI } \\
(\mu \mathrm{m})\end{array}$ & $\begin{array}{c}\text { BII } \\
(\mu \mathrm{m})\end{array}$ & BAR & $\begin{array}{c}\text { OPX } \\
(\%)\end{array}$ \\
\hline (1917) Cuyo & 0.93 & 1.95 & $0.670 \pm 0.1526$ & 33.28 \\
(8567) 1996 HW1 & 0.99 & 2.06 & $0.485 \pm 0.2687$ & 25.50 \\
(16960) 1998 QS52 & 0.97 & 2.03 & $0.232 \pm 0.1996$ & 14.94 \\
\hline
\end{tabular}

Notes. The estimation error for band centers (BI, BII) is \pm 0.005 .

submicroscopic single-domain metallic Fe (4-30 nm), produced in the space environment by a reduction of $\mathrm{FeO}$ in minerals. Referred to as nanophase reduced iron - " $n p F e^{0 ",}$, these are formed through the fractional processes that occur during ionparticle sputtering, vapor deposits from energetic micromete-

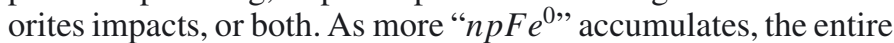
continuum becomes redder until it is almost linear through to the near-infrared region. With small amounts of " $n p F e e^{0}$, , reddening of only the visible region of the spectra occur (Pieters et al. 2000).

A space weathering model has been applied to five spectra in our samples (of S-type objects). For two asteroids, (188452) 2004 HE62 and 2010 TD554, the models imply that the iron content ambiguity changes the best analog among meteorite samples. Thus, the best mineralogical analog will always be an OC meteorite, the same petrologic type, but the spectra for a sample containing Fe will be different. This could be explained in the following terms: highly curved continua occur for samples with small amounts of $n p F e^{0}$, and the more linear continua occur for samples with large amounts of $n p F e^{0}$ (Pieters et al. 2000).

A quantitative comparison between the reflectance properties of (1917) Cuyo, (8567) 1996 HW1, and (16960) 1998 QS52 (since for these objects we have both visible and NIR data) and potential meteorite analogs could be made with the parameters computed from the model of Cloutis et al. (1986). The values of these parameters are given in Tabel 5. Plotting Band I center versus the BAR (Gaffey et al. 1993), we found 
that all three objects are located in the ordinary chondrite region (Fig. 5). (1917) Cuyo and (16960) 1998 QS52 are under the olivine-orthopyroxene mixing line, while (8567) $1996 \mathrm{HW} 1$ is above the olivine-orthopyroxene mixing line.

Another comparison was made by plotting the Band I center versus the Band II center (Fig. 5). Considering the results of de León et al. (2010), we found that (1917) Cuyo is in the region of OC -H meteorites, while 16960 is in the region of OC-L meteorites. (8567) 1996 HW1 is outside the enclosed areas, between the regions for $\mathrm{L}$ and LL chondrites.

This statistical interpretation of the results agrees with our results obtained by comparison to Relab meteorite spectra. These measured parameters are direct indications of a spectrum's basic properties - revealing their distributions without making any assumptions about their underlying mineralogy (Vernazza et al. 2008).

\section{Conclusions}

We obtained NIR spectra for eight near-Earth asteroids using IRTF/SpeX. The observations were performed remotely between the IRTF and Observatoire de Paris-Meudon. This technique proved to be robust while providing full control of the spectrograph and access to several telescope operations (focus, tracking, etc.).

Four of the observed objects have $\Delta V$ lower than $7 \mathrm{~km} \mathrm{~s}^{-1}$, which make them suitable targets in terms of propulsion for a future spacecraft mission.

We have modelled and interpreted the obtained spectra using a variety of techniques with the goal of interpreting the asteroidal surfaces in terms of their mineralogical composition. Each asteroid spectrum was analyzed to obtain its taxonomic class and the closest matching meteoritic analogs in the laboratory. We have considered and discussed the effects of space weathering based on the model of Brunetto et al. (2006).

The taxonomic classification of five objects was reviewed and we assigned a corresponding type to the other three asteroids that had not been previously classified. We found that (1917) Cuyo, (8567) 1996 HW1, (16960) 1998 QS52, (188452) 2004 HE62, and 2010 TD54 are in the S-complex. For these objects, a good matching with the spectra of ordinary chondrites meteorites has been obtained.

The asteroid Jasonwheeler was found to have a NIR spectrum similar to that of carbonaceous chondrite meteorites. It was classified to be between D and T taxonomic class. Since these classes are considered to have primitive compositions and the $\Delta V$ for this object is smaller than $7 \mathrm{~km} \mathrm{~s}^{-1}$, Jasonwheeler could be a very interesting candidate for a sample and return spacecraft mission.

Acknowledgements. The article is based on observations acquired with InfraRed Telescope Facilities as well as the CODAM remote facilities. We thank all the telescope operators for their contribution. This research utilizes spectra acquired with the NASA RELAB facility at Brown University.

The work of Marcel Popescu was supported by the ESA traineeships program Ref: RES-HM/1636.

The work of Dan Alin Nedelcu was partially supported by the Romanian National Authority for Scientific Research (ANCS) under the project POSCCEA2-O2.1.2-2009-2/651.

\section{References}

Barucci, M. A., Capria, M. T., Coradini, A., \& Fulchignoni, M. 1987, Icarus, 72, 304

Binzel, R. P., Lupishko, D., di Martino, M., Whiteley, R. J., \& Hahn, G. J. 2002, Asteroids III, 255

Binzel, R. P., Perozzi, E., Rivkin, A. S., et al. 2004a, Meteoritics and Planetary Science, 39, 351

Binzel, R. P., Rivkin, A. S., Stuart, J. S., et al. 2004b, Icarus, 170, 259

Binzel, R. P., Morbidelli, A., Merouane, S., et al. 2010, Nature, 463, 331

Birlan, M., Barucci, M. A., \& Fulchignoni, M. 1996, A\&A, 305, 984

Birlan, M., Barucci, M. A., Vernazza, P., et al. 2004, New Astron., 9, 343

Birlan, M., Vernazza, P., Fulchignoni, M., et al. 2006, A\&A, 454, 677

Birlan, M., Vernazza, P., \& Nedelcu, D. A. 2007, A\&A, 475, 747

Birlan, M., Binzel, R. P., Nedelcu, D., et al. 2009, in AAS/Division for Planetary Sciences Meeting Abstracts, 41, 34.08

Brunetto, R., \& Strazzulla, G. 2005, Icarus, 179, 265

Brunetto, R., Vernazza, P., Marchi, S., et al. 2006, Icarus, 184, 327

Bus, S. J., \& Binzel, R. P. 2002, Icarus, 158, 146

Clark, B. E., Hapke, B., Pieters, C., \& Britt, D. 2002, in Asteroids III, ed. W. F. Bottke Jr., A. Cellino, P. Paolicchi, \& P. Binzel (University of Arizona Press: Tucson), 585

Cloutis, E. A., Gaffey, M. J., Jackowski, T. L., \& Reed, K. L. 1986, J. Geophys. Res., 91, 641

Das Gupta, S. P., Sen Gupta, P. R., Dube, A., Sen Gupta, N. R., \& Das Gupta, D. R. 1978, Mineralogical Magazine, 42, 493

de León, J., Licandro, J., Serra-Ricart, M., Pinilla-Alonso, N., \& Campins, H. 2010, A\&A, 517, A23

de León, J., Mothé-Diniz, T., Licandro, J., Pinilla-Alonso, N., \& Campins, H. 2011, A\&A, 530, L12

DeMeo, F. E., Binzel, R. P., Slivan, S. M., \& Bus, S. J. 2009, Icarus, 202, 160

Dobrica, E. 2010, Ph.D. Thesis, University Paris Sud

Durkee, I. R. 2010, Minor Planet Bulletin, 37, 18

Fornasier, S., Barucci, M. A., Binzel, R. P., et al. 2003, A\&A, 398, 327

Fulchignoni, M., Birlan, M., \& Antonietta Barucci, M. 2000, Icarus, 146, 204

Gaffey, M. J., Burbine, T. H., Piatek, J. L., et al. 1993, Icarus, 106, 573

Gradie, J., \& Veverka, J. 1986, Icarus, 66, 455

Hapke, B. 2001, J. Geophys. Res., 106, 10039

Hicks, M., \& Rhoades, H. 2010, The Astronomer's Telegram, 2984, 1

Higgins, D., Pravec, P., Kusnirak, P., et al. 2006, Minor Planet Bulletin, 33, 8

Kong, P., \& Ebihara, M. 1997, Geochim. Cosmochim. Acta, 61, 2317

Loeffler, M. J., Dukes, C. A., \& Baragiola, R. A. 2009, J. Geophys. Res. (Planets), 114, E03003

Luu, J. X., \& Jewitt, D. C. 1990, AJ, 99, 1985

Michel, P., \& Delbo, M. 2010, Icarus, 209, 520

Michelsen, R., Nathues, A., \& Lagerkvist, C. 2006, A\&A, 451, 331

Milani, A., Chesley, S. R., \& Valsecchi, G. B. 2000, Planet. Space Sci., 48, 945

Morbidelli, A., Jedicke, R., Bottke, W. F., Michel, P., \& Tedesco, E. F. 2002, Icarus, 158, 329

Mueller, M., Delbo', M., Hora, J. L., et al. 2011, AJ, 141, 109

Nedelcu, D. A. 2010, Ph.D. Thesis, Observatoire de Paris

Pieters, C. M., Taylor, L. A., Noble, S. K., et al. 2000, Meteor. Planet. Sci., 35 , 1101

Popescu, M., \& Birlan, M. 2011, unpublished

Rayner, J. T., Toomey, D. W., Onaka, P. M., et al. 2003, PASP, 115, 362

Rivkin, A. S., Binzel, R. P., Sunshine, J., et al. 2004, Icarus, 172, 408

Rivkin, A. S., Binzel, R. P., \& Bus, S. J. 2005, Icarus, 175, 175

Taylor, P. A., Howell, E. S., Magri, C., et al. 2009, in AAS/Division for Planetary Sciences Meeting Abstracts, 41, 32.01

Tholen, D. J. 1984, Ph.D. Thesis, Arizona Univ., Tucson

Tody, D. 1986, in SPIE Conf. Ser. 627, ed. D. L. Crawford, 733

Vacca, W. D., Cushing, M. C., \& Rayner, J. T. 2004, PASP, 116, 352

Vander Haagen, G. A. 2011, Minor Planet Bulletin, 38, 10

Vernazza, P. 2006, Ph.D. Thesis, Observatoire de Paris

Vernazza, P., Binzel, R. P., Thomas, C. A., et al. 2008, Nature, 454, 858

Vernazza, P., Binzel, R. P., Rossi, A., Fulchignoni, M., \& Birlan, M. 2009, Nature, 458, 993

Veverka, J., Robinson, M., Thomas, P., et al. 2000, Science, 289, 2088

Warner, B. D. 2009, Minor Planet Bulletin, 36, 7

Wisniewski, W. Z., Michalowski, T. M., Harris, A. W., \& McMillan, R. S. 1997, Icarus, 126, 395

Zellner, B., Tholen, D. J., \& Tedesco, E. F. 1985, Icarus, 61, 355

Pages 12 to 15 are available in the electronic edition of the journal at http://www . aanda.org 


\section{Appendix A: Asteroid spectra vs meteorites spectra}

The closest spectral matches between the reflectance spectra of the asteroids analyzed in our article and the laboratory spectra of different meteorites. Additional details related to meteorites samples are given in Table 3.

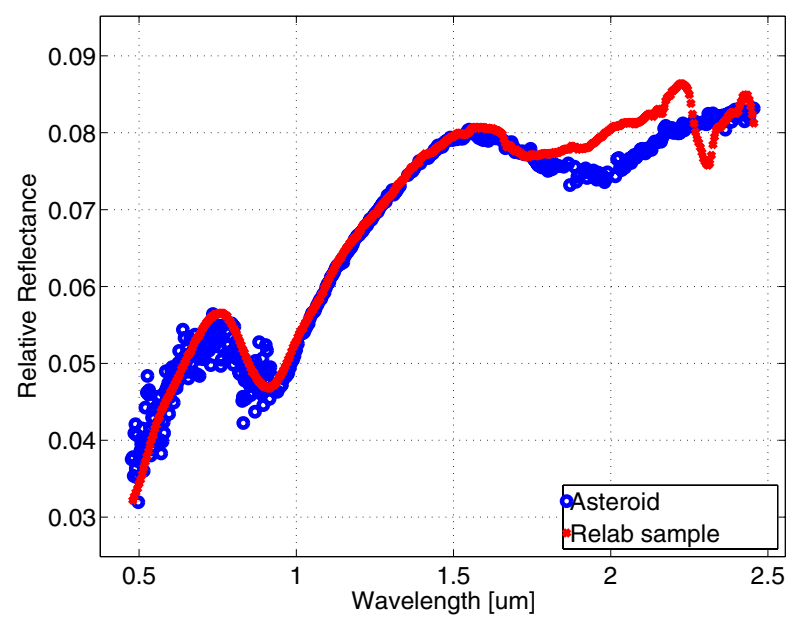

Fig. A.1. Reflectance spectrum of (1917) Cuyo and the closest match resulting from meteorite comparison - H3-4 ordinary chondrite Dhajala.

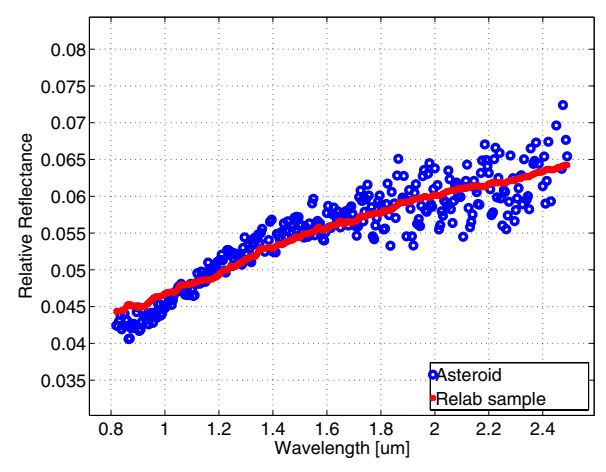

(a)

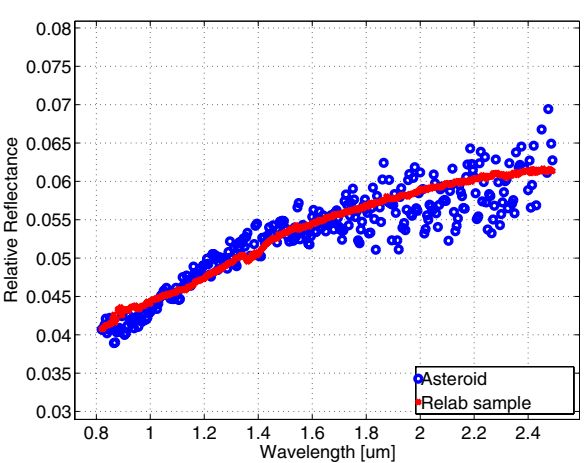

(b)

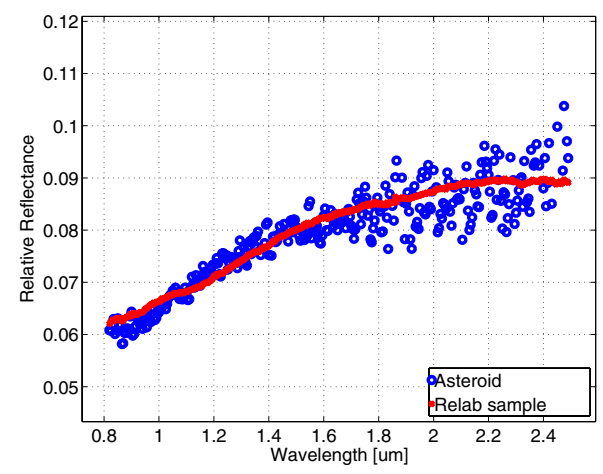

(c)

Fig. A.2. Reflectance spectrum of (5620) Jasonwheeler and the closest three matches resulting from meteorite comparison: a) CM2 carbonaceous chondrite Mighei/Meghei; b) CM2 carbonaceous chondrite Cold Bokkeveld; c) CM2 carbonaceous chondrite ALH84029.

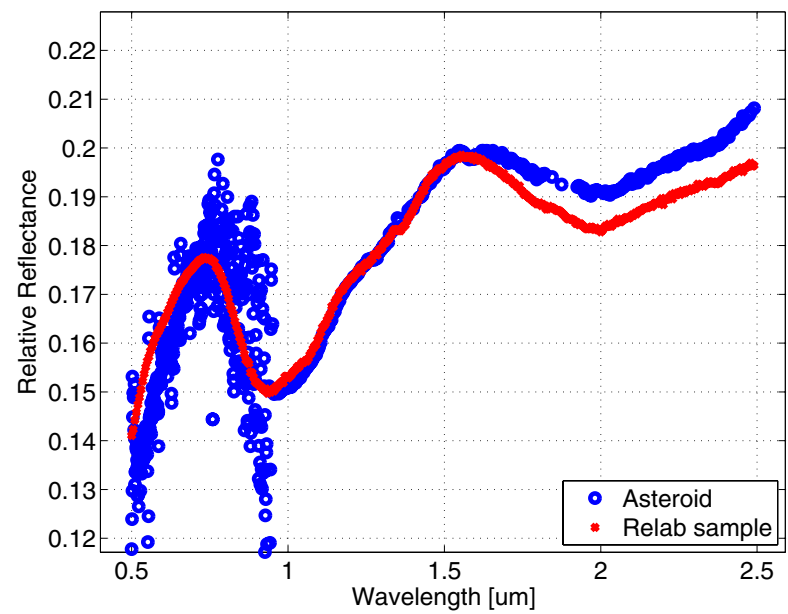

Fig. A.3. Reflectance spectrum of (8567) 1996 HW1 and the closest match resulting from the meteorite comparison - LL4 ordinary chondrite Hamlet. 
M. Popescu et al.: Spectral properties of eight Near-Earth Asteroids

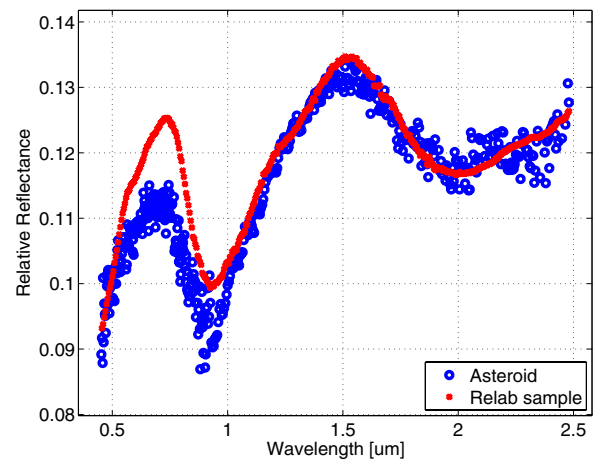

(a)

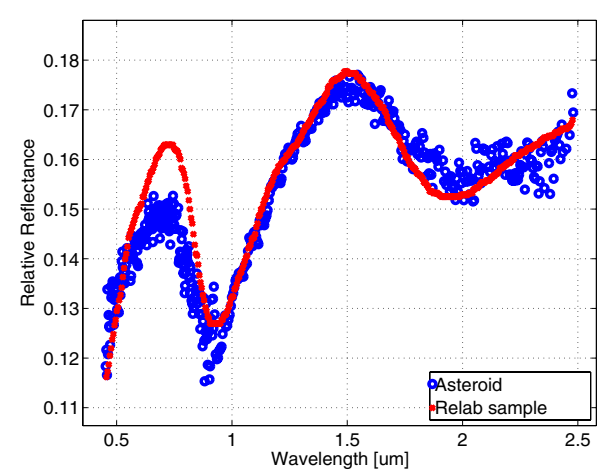

(b)

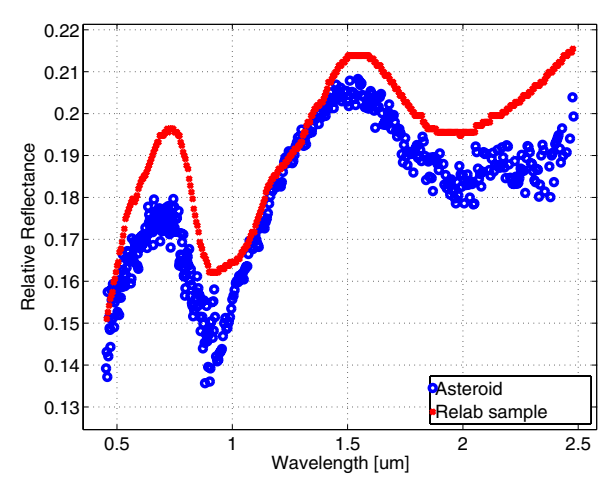

(c)

Fig. A.4. Reflectance spectrum of (16960) 1998 QS52 and the closest three matches resulting from a meteorite comparison: a) L4 ordinary chondrite Saratov; b) L5 ordinary chondrite Homestead; c) LL4 ordinary chondrite Hamlet.

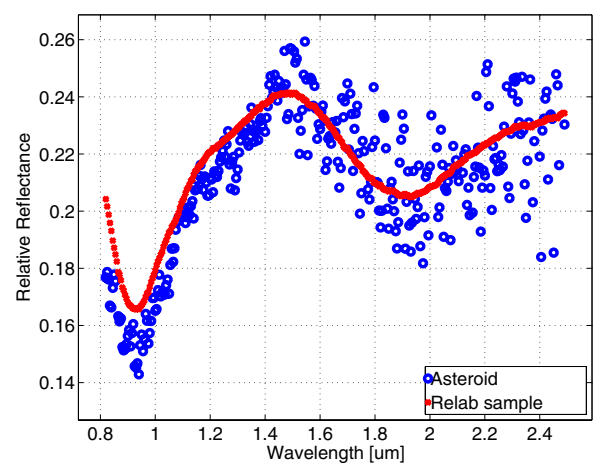

(a)

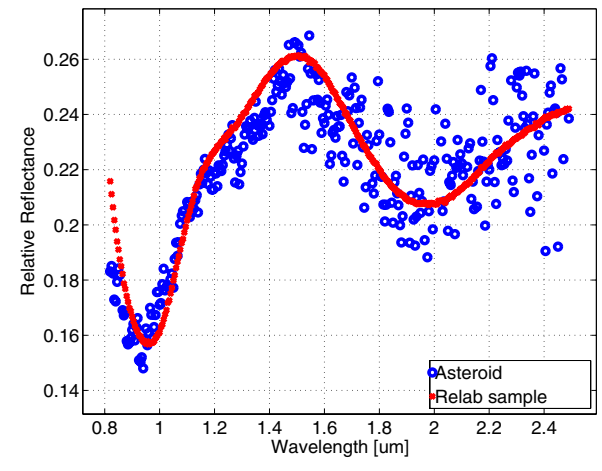

(b)

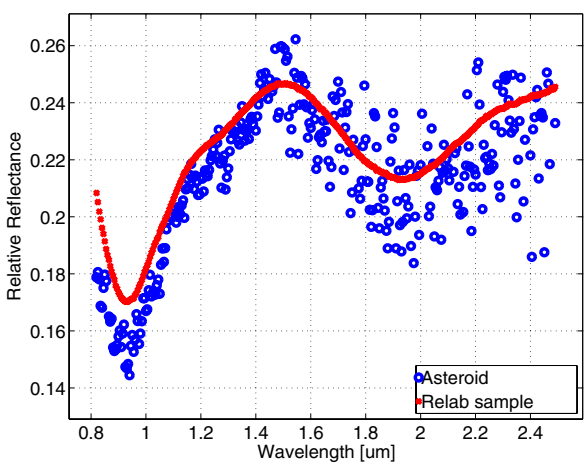

(c)

Fig. A.5. Reflectance spectrum of (188452) 2004 HE62 and the closest three matches resulting from meteorite comparison: a) L6 ordinary chondrite La Criolla; b) LL6 ordinary chondrite Cherokee Springs; c) L6 ordinary chondrite Wold Cottage.

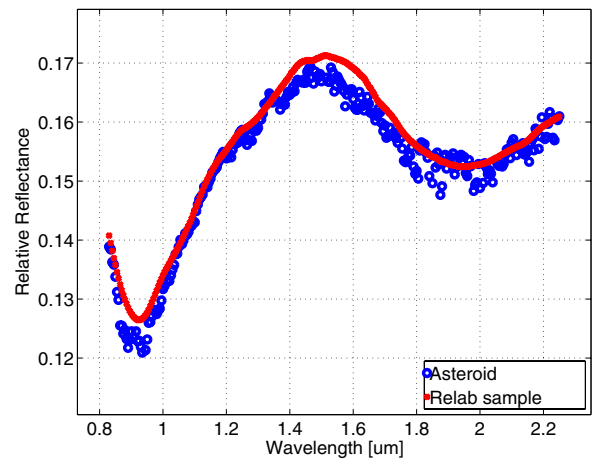

(a)

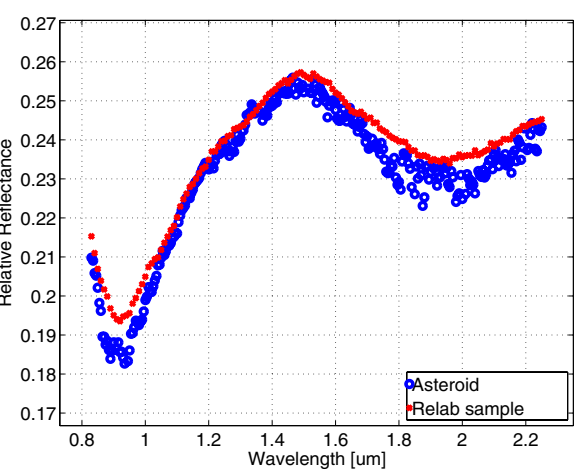

(b)

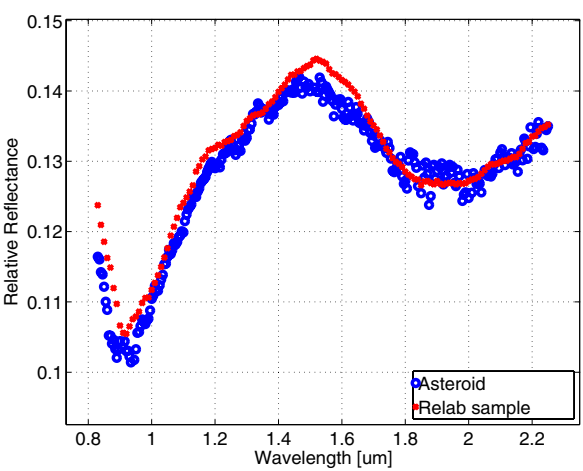

(c)

Fig. A.6. Reflectance spectrum of (188452) 2004 HE62 and the closest three matches resulting from meteorite comparison: a) L4 ordinary chondrite Saratov; b) L5 ordinary chondrite Mirzapur; c) L4 ordinary chondrite Rio Negro. 


\section{Appendix B: De-reddened asteroid spectra vs meteorites spectra}

The closest spectral matches between the de-reddened spectra of the S-type asteroids analyzed in our article and the laboratory spectra of different meteorites. Additional details related to meteorites samples are given in Table 3.

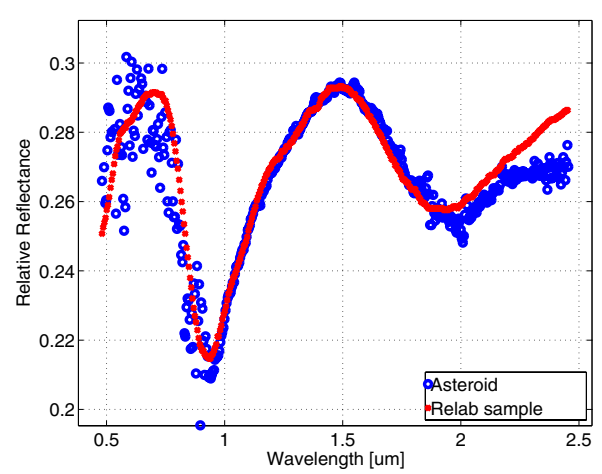

(a)

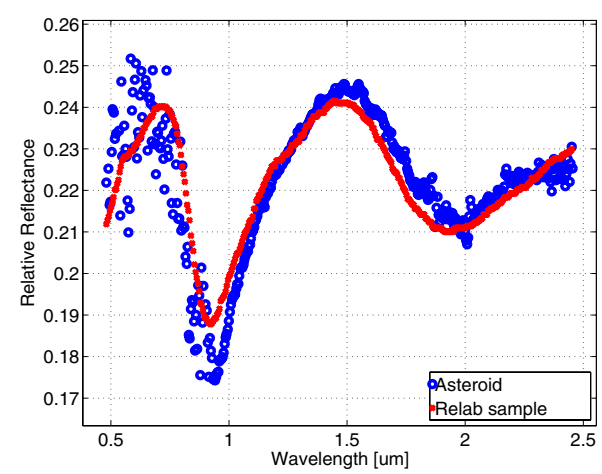

(b)

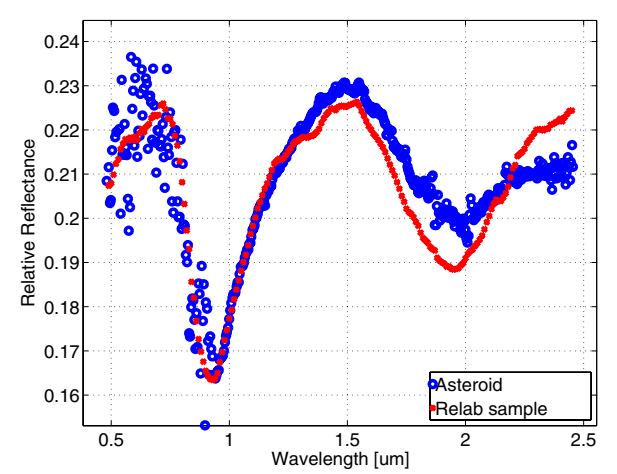

(c)

Fig. B.1. De-reddened spectrum of (1917) Cuyo and the closest three matches resulting from meteorite comparison: a) H6 ordinary chondrite Lancon; b) H5 ordinary chondrite Collescipoli; c) H5 ordinary chondrite Ehole.

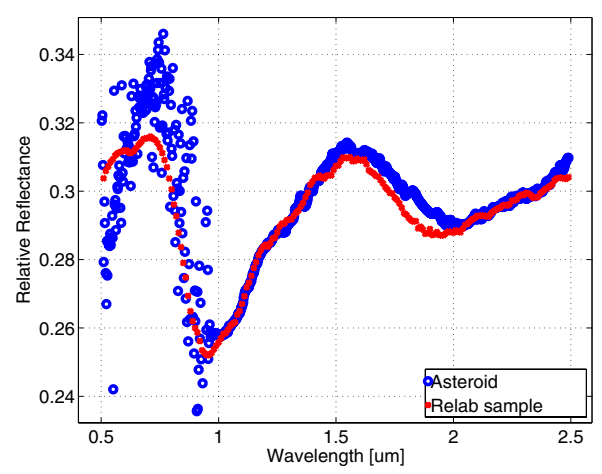

(a)

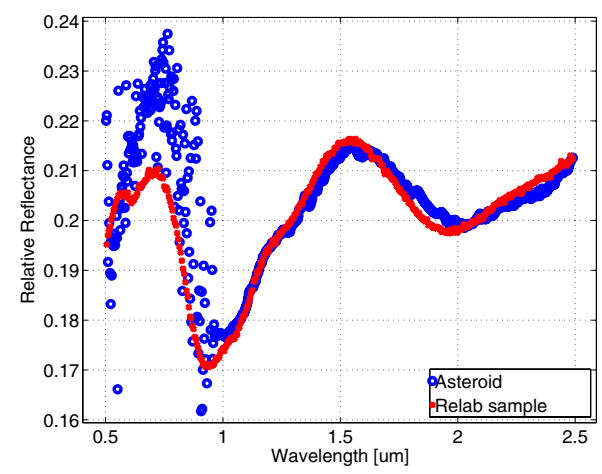

(b)

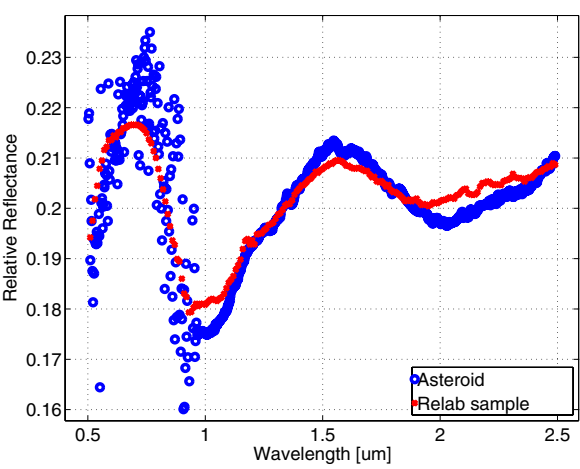

(c)

Fig. B.2. De-reddened spectrum of (8567) 1996 HW1 and the closest three matches resulting from meteorite comparison: a) LL6 ordinary chondrite Cherokee Springs; b) L3-6 ordinary chondrite Hedjaz; c) LL6 ordinary chondrite Ensisheim.

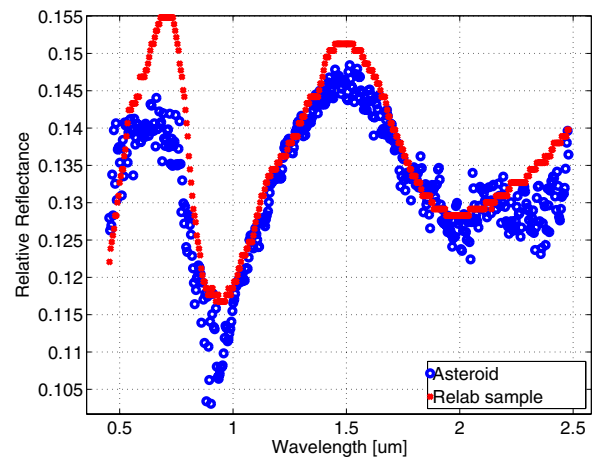

(a)

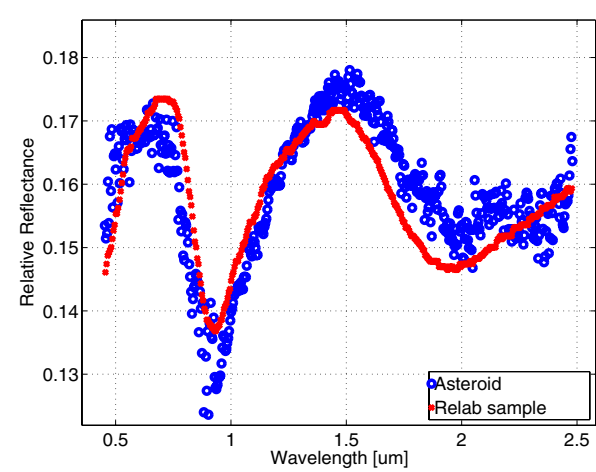

(b)

Fig. B.3. De-reddened spectrum of (16960) 1998 QS52 and the closest two matches resulting from meteorite comparison: a) LL4 ordinary chondrite Hamlet; b) $\mathrm{H} 4$ ordinary chondrite Gruneberg. 
M. Popescu et al.: Spectral properties of eight Near-Earth Asteroids

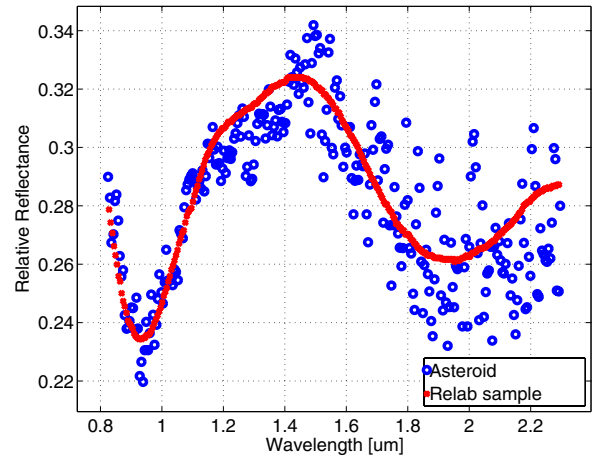

(a)

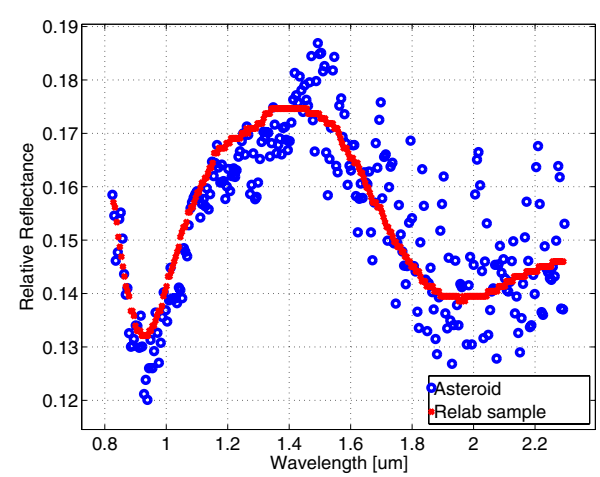

(b)

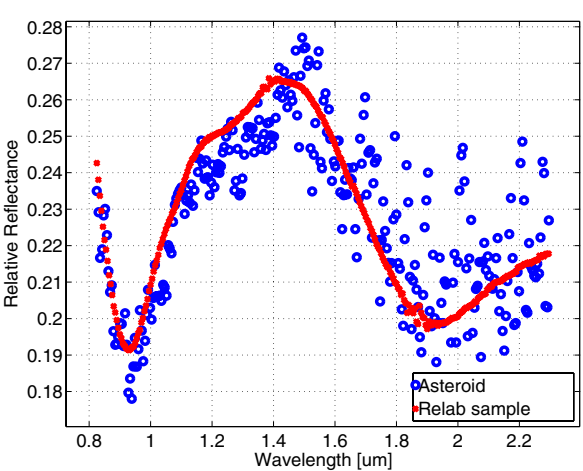

(c)

Fig. B.4. De-reddened spectrum of (188452) 2004 HE62 and the closest three matches resulting from meteorite comparison: a) H6 ordinary chondrite Nanjemoy; b) H5 ordinary chondrite Olmedilla de Alarcon; c) H5 ordinary chondrite MAC88119.9.

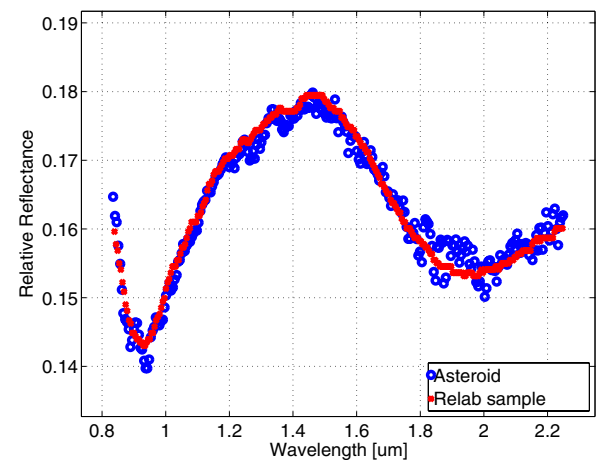

(a)

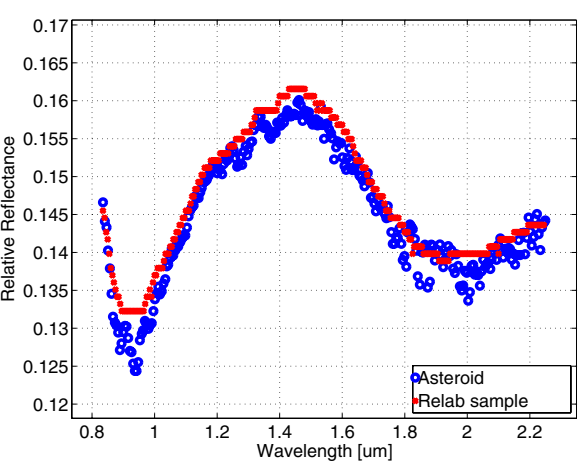

(b)

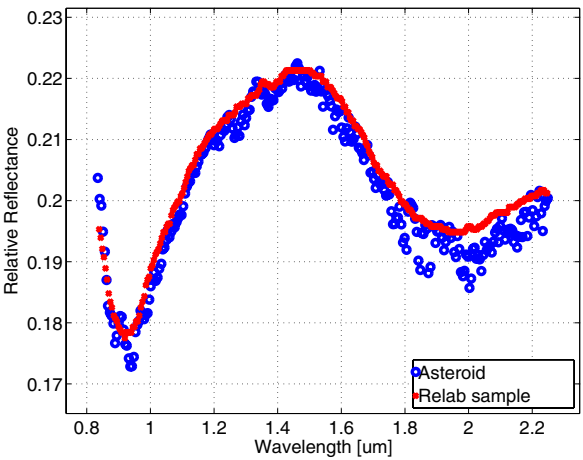

(c)

Fig. B.5. De-reddened spectrum of 2010 TD54 and the closest three matches resulting from meteorite comparison: a) H4 ordinary chondrite Gruneberg; b) H6 ordinary chondrite Queen's Mercy; c) H4 ordinary chondrite Ochansk. 